\title{
1 Stable Engraftment of a Human Gut Bacterial Microbiome in Double Humanized BLT-mice
}

2 Lance Daharsh ${ }^{1,2}$, Amanda E. Ramer-Tait ${ }^{3}$, * Qingsheng $\mathrm{Li}^{1,2}$

3

$4{ }^{1}$ Nebraska Center for Virology, ${ }^{2}$ School of Biological Sciences, ${ }^{3}$ Department of Food Science and

5 Technology, University of Nebraska-Lincoln, Lincoln, NE 68583, USA

$7 \quad$ Corresponding author: Qingsheng Li (qli@unl.edu)

\section{Abstract}

\section{Background}

11 Humanized mice featuring a functional human immune system are an important pre-clinical

12 model for examining immune responses to human-specific pathogens. This model has been

13 widely utilized to study human diseases that are otherwise impossible or difficult to investigate

14 in humans or with other animal models. However, one limitation of using humanized mice is

15 their native murine gut microbiome, which significantly differs from the one found in humans.

16 These differences may be even greater for mice housed and bred in specific pathogen free

17 conditions. Given the importance of the gut microbiome to human health and disease, these

18 differences may profoundly impact the ability to translate the results from humanized mice

19 studies to human disease. Further, there is a critical need for improved pre-clinical models to

20 study the complex in vivo relationships of the gut microbiome, immune system, and human

21 disease. We therefore created double humanized mice with both a functional human immune

22 system and stable human-like gut microbiome. 


\section{Results}

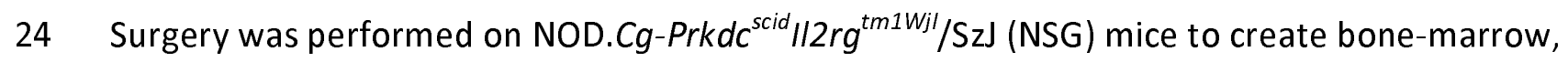

25 liver, thymus (BLT) humanized mice. After immune reconstitution, mice were treated with

26 broad spectrum antibiotics to deplete murine gut bacteria and then transplanted with fecal

27 material from healthy human donors. Characterization of 173 fecal samples obtained from 45

28 humanized mice revealed that double humanized mice had unique 16S rRNA gene profiles

29 consistent with those of the individual human donor samples. Importantly, transplanted

30 human-like gut microbiomes were stable in mice for the duration of the study, up to 14.5 weeks

31 post-transplant. Microbiomes of double humanized mice also harbored predicted functional

32 capacities that more closely resembled those of the human donors compared to humanized

33 mice.

\section{Conclusions}

35 Here, we describe successful engraftment of a stable human microbiome in BLT humanized

36 mice to further improve this preclinical humanized mouse model. These double humanized

37 mice represent a unique and tractable new model to study the complex relationships between

38 the human gut microbiome, human immune system, and human disease in vivo.

Background

The complex ecosystem of the gut microbiome plays a critical role in human health and

42 disease [1-5]. Specifically, the gut microbiome has a highly reciprocal and dynamic relationship

43 with the immune system. Antigens derived from the gut microbiome influence host immune

44 responses, and the immune system in turn contributes to shaping the spatial distribution and 
45 composition of the gut microbiota [6-8]. Humanized mice (hu-mice) with an engrafted human

46 immune system have facilitated important advancements in the study of human cancer,

47 autoimmune diseases, hematopoiesis, and infectious diseases [9-17]. However, gut

48 microbiomes of hu-mice are murine in origin and are often not well-characterized in

49 translational studies. The murine gut microbiome differs substantially in composition and

50 function from that of humans [18], primarily due to anatomical differences as well as other

51 factors such as diet [19]. Considering the importance of the gut microbiota to proper

52 immunological development and influencing immune responses, the murine origin of the

53 microbiome harbored by hu-mice could affect translational study outcomes. Consequently, a

54 need exists to not only characterize the gut microbiomes of hu-mice, but also impart these mice

55 with a more human-like gut microbiome to improve the translational aspects of the model for

56 human medicine.

57 The creation of a new pre-clinical hu-mice model to study the human immune in the

58 context of a human microbiome offers numerous benefits over existing options. Many aspects

59 of human disease are difficult or impossible to study directly in humans due to practical or

60 ethical concerns. Non-human primate models are informative but are genetically outbred, and

61 large studies are often resource and cost prohibitive. Many important discoveries in the

62 microbiome field have been made using mouse models; however, translating results from

63 mouse studies to humans has often proved difficult. The use of germ-free mice reconstituted

64 with human-like gut microbiomes has been the gold standard in studying the relationship of the

65 gut microbiome to human health and disease $[20,21]$. However, working with or deriving germ-

66 free animals requires expertise and facilities that are not always available. Further, many 
67 immunodeficient mouse strains commonly used to reconstitute a human immune system, such

68 as NOD.Cg-Prkdc $c^{\text {scid }} / 12 \mathrm{rg}^{\mathrm{tm} 1 \mathrm{~W}_{\mathrm{Wl}}} / \mathrm{SzJ}$ (NSG), are currently not commercially available as germ-free.

69 We therefore created a double hu-mice model featuring both a functional human immune

70 system and a stable human-like gut microbiome under specific pathogen free (SPF) conditions.

71 Here, we show that double hu-mice had unique 16S rRNA gene profiles based on the individual

72 human donor sample with which they were colonized. Importantly, the transplanted human-

73 like microbiome was stable in the mice for the duration of the study, up to 14.5 weeks post-

74 transplant. Double hu-mice also harbored gut microbiomes with a more human-like predicted

75 functional capacity compared to their hu-mice counterparts.

\section{Results}

78 Gut microbiomes of double hu-mice are distinct and more human-like compared to hu-mice.

79 To create double hu-mice, surgery was performed on NSG mice to create bone-marrow,

80 liver, thymus (BLT) hu-mice. Hu-mice were then pre-treated with a cocktail of broad-spectrum

81 antibiotics and administered fecal transplants using fecal material from healthy human donors

82 (see Methods and Daharsh et al.[22] for detailed descriptions and demonstrations). Multiple

83 cohorts of double hu-mice were created using fecal material from one of three unique healthy

84 human donors or an equal mixture of all three (Table 1). We used 16S rRNA gene sequencing

85 and characterized the gut bacterial microbiome of 100 fecal samples from 16 double hu-mice

86 and compared them with 67 fecal samples representing the pre-existing murine gut bacterial

87 microbiomes of hu-mice and the profiles of the 4 human fecal donor samples. To visualize beta-

88 diversity relationships between hu-mice, double hu-mice, and the human donor samples, non- 
89 metric multidimensional scaling (NMDS) and principal coordinate analysis (PCOA) plots were

90 created (Fig. 1). Using NMDS, the gut microbiome profiles of the three groups separated into

91 distinct clusters (Fig. 1a). Using PCoA, the human donor samples fell within the double hu-mice

92 hull, which was distinct from the hu-mice profiles (Fig. 1b). Both dimensionality reduction

93 methods showed that after engraftment of a human gut microbiome, double hu-mice

94 represented a distinct population that clustered closer to the human donor samples compared

95 to hu-mice harboring murine gut microbiomes. Importantly, there was no reversion to hu-mice

96 profiles post-transplant. Displaying the relatedness of the samples through a hierarchical

97 dendrogram based on Bray-Curtis distances further confirmed the similarity of double hu-mice

98 microbiomes to the human donor samples and differentiated them from those of hu-mice (Fig.

$992)$.

100 Table 1. Experimental summary of double hu-mice cohorts

\begin{tabular}{|c|c|c|c|c|c|c|c|c|c|}
\hline Cohort & $\begin{array}{c}\text { Antibiotic } \\
\text { start }\end{array}$ & $\begin{array}{c}\text { Antibiotic } \\
\text { duration }\end{array}$ & $\begin{array}{c}\text { Number of } \\
\text { fecal } \\
\text { transplants }\end{array}$ & $\begin{array}{c}\text { Human fecal } \\
\text { donor }\end{array}$ & $\begin{array}{c}\text { Maximum } \\
\text { weeks } \\
\text { post- } \\
\text { transplant }\end{array}$ & $\begin{array}{l}\text { Double } \\
\text { hu-mice }\end{array}$ & $\begin{array}{c}\text { Double } \\
\text { hu-mice } \\
\text { fecal } \\
\text { samples }\end{array}$ & $\begin{array}{l}\mathrm{Hu}- \\
\text { mice }\end{array}$ & $\begin{array}{c}\text { Hu- } \\
\text { mice } \\
\text { fecal } \\
\text { samples }\end{array}$ \\
\hline $\begin{array}{l}\text { Antibiotic } \\
\text { Pilot }\end{array}$ & $\begin{array}{l}\text { After immune } \\
\text { reconstitution }\end{array}$ & 9 days & 0 & NA & 0 & 0 & 0 & 6 & 9 \\
\hline $\begin{array}{l}\text { Donor } 65 \\
\text { Cohort } 1\end{array}$ & $\begin{array}{l}\text { After immune } \\
\text { reconstitution }\end{array}$ & 14 days & $\begin{array}{c}2 \text { (24\& } 48 \\
\text { hr post } \\
\text { antibiotics) }\end{array}$ & 65 & 13.5 & 3 & $35 \#$ & 3 & 23 \\
\hline $\begin{array}{l}\text { Donor } 65 \\
\text { Cohort } 2\end{array}$ & $\begin{array}{l}\text { After immune } \\
\text { reconstitution }\end{array}$ & 14 days & $\begin{array}{c}2 \text { (24 \& } 48 \\
\text { hr post } \\
\text { antibiotics) }\end{array}$ & 65 & 14.5 & 2 & $16 \#$ & 8 & 8 \\
\hline Donor 74 & $\begin{array}{l}\text { After immune } \\
\text { reconstitution }\end{array}$ & 7 days & $\begin{array}{c}2 \text { (24 \& } 48 \\
\text { hr post } \\
\text { antibiotics) }\end{array}$ & 74 & 6 & 3 & $17 \#$ & 3* & 3* \\
\hline
\end{tabular}




\begin{tabular}{|c|c|c|c|c|c|c|c|c|c|}
\hline Donor 82 & $\begin{array}{l}\text { After immune } \\
\text { reconstitution }\end{array}$ & 7 days & $\begin{array}{c}2 \text { (24 \& } 48 \\
\text { hr post } \\
\text { antibiotics) }\end{array}$ & 82 & 6 & 4 & $27 \#$ & $3^{*}$ & 3* \\
\hline Donor Mix & $\begin{array}{l}3 \text { days after } \\
\text { hu-mice } \\
\text { surgery }\end{array}$ & 14 days & $\begin{array}{c}2 \text { (24\& } \& 48 \\
\text { hr post } \\
\text { antibiotics) }\end{array}$ & $\begin{array}{c}\text { Equal } \\
\text { mixture of } \\
65,72, \& 84\end{array}$ & 9 & 4 & $21 \#$ & 9 & 10 \\
\hline
\end{tabular}

101

We also observed that double hu-mice maintained the pre-existing relationships

103 between gut microbiome profiles of the human fecal donors (Fig. 1c \& 1d). Importantly, pre-

104 treatment samples from corresponding double hu-mice in each cohort had similar gut

105 microbiome profiles as untreated control hu-mice. After engraftment, double hu-mice

106 resembled the individual human donor that was transplanted as demonstrated by the

107 relationships between the human donor microbiome profiles. Human donors 72 and 84 had

108 more similar profiles to one another than to human donor 65 . This relationship was maintained

109 in the double hu-mice after engraftment. We also prepared an "un-biased" human sample by

110 mixing equal parts of the three human donor fecal samples, designated hereafter as donor mix.

111 The microbiome profile of this mixed sample resembled a mixture of the three individual

112 human donor profiles. Specifically, the mixed donor sample more closely resembled individual

113 donors 72 and 84 , and this observation was mirrored in the double hu-mice engrafted with the

114 donor mix sample.

115 We also investigated the impact of antibiotic treatment duration on the engraftment of

116 human gut microbiome. The double hu-mice engrafted with human donors 74 and 82 were

117 generated after only 7 days of antibiotic pre-treatment. Their microbiome profiles were less

118 similar to the human donor profiles than those from cohorts pre-treated with antibiotics for 14 
119 days prior to fecal transplant (Fig. 1c \& 1d). We found the 2 weeks of antibiotic treatment was

120 optimal for the creation of double hu-mice. Together, these results demonstrate that our

121 approach to generating double hu-mice is reproducible and able to create hu-mice with unique

122 16S rRNA gene profiles based on the individual human fecal donor.

124 Gut microbiomes of double hu-mice have increased levels of alpha diversity compared to hu-

125 mice.

127 hypothesized that highly immunodeficient mice, such as NSG, would have low pre-existing gut

128 microbiome diversity, especially when the mice were housed under SPF conditions with limited

129 exposure to outside sources of microbes. We tested this hypothesis and found that the gut

130 microbial diversity of double hu-mice with a functional immune system significantly increased

131 to the levels observed in our human donor samples compared to hu-mice. Several alpha

132 diversity measurements confirmed that hu-mice had very low measures of alpha diversity

133 compared to our human donor samples (Fig. 3). However, after engraftment, double hu-mice

134 had increased species richness compared to hu-mice $(P<.001)$ and did not differ significantly

135 from the human donor samples (Fig. 3a). Further, the Shannon index values of the human

136 donor samples were significantly higher than hu-mice $(P<.05)$ but were not significantly

137 different from the double hu-mice samples. Double hu-mice had increased Simpson index

138 values compared to hu-mice, but the human donor samples were significantly higher than both

139 the double hu-mice $(\mathrm{P}<.05)$ and hu-mice $(\mathrm{P}<.05)$. As expected, samples taken during antibiotic

140 treatment had the lowest measures of all three diversity metrics tested. Alpha diversity metrics 
141 were also measured based on the human donor sample engrafted (Supplemental Figure -

142 Alpha_Diversity.pdf). Double hu-mice engrafted after 14 days of antibiotics (donor 65 cohorts 1

$143 \& 2$, donor mix) had higher levels of alpha diversity compared to double hu-mice engrafted

144 after only 7 days of antibiotics (donors $74 \& 82$ ). Overall, double hu-mice had increased alpha

145 diversity compared to hu-mice that was more similar to the levels observed in the human donor

146 samples. The shorter antibiotic treatment duration (7 days versus 14 days) was associated with

147 lower alpha diversity measurements in double hu-mice engrafted with human donors 74 or 82.

The relative abundance of gut bacteria in double hu-mice is similar to that found in human

150 donor samples.

We next hypothesized that if bacteria from the human donors were successfully

152 engrafted into double hu-mice, then we would see more taxonomic similarities between human

153 donor gut microbiome profiles and double hu-mice versus hu-mice. Several differences were

154 observed in the relative abundances of double hu-mice based on the length of antibiotic

155 treatment, the human fecal donor sample engrafted, and mouse cohort (Supplemental Figure -

156 Relative_Abundances.pdf). At the Phylum level, both double hu-mice and hu-mice samples

157 were largely represented by Actinobacteria, Bacteroidetes, Firmicutes, and Verrucomicrobia.

158 Interestingly, we found that hu-mice had high proportions of Verrucomicrobia that remained

159 high even after antibiotic treatment and engraftment with human donor samples that had low

160 abundances of Verrucomicrobia. At the Family level, hu-mice samples had a higher relative

161 abundance of S24-7 than double hu-mice and human donor samples (Fig. 4). Both hu-mice and

162 double hu-mice samples had higher relative abundances of Lactobacillaceae and 
163 Verrucomicrobiaceae than human donor samples. The human donor and double hu-mice

164 samples had higher relative abundances of Bacteroidaceae than hu-mice samples. The human

165 donor samples also had a much higher relative abundance of Lachnospiraceae.

166 We next compared the taxonomic differences between the microbiomes using Kruskal-

167 Wallis testing with FDR adjusted P values below .05 (Supplemental File - KW_Testing.xlsx). We

168 found 195 significant differences between the hu-mice and human donor samples and 170

169 significant differences between hu-mice and double hu-mice. However, only 108 significant

170 differences were observed between double hu-mice and human donor samples. Both the

171 human donor and double hu-mice samples had significantly higher relative abundances of the

172 genus Bacteroides, while hu-mice had a significantly higher relative abundance of the family

173 S24-7. Human donor samples had a higher relative abundance of the Phylum Firmicutes

174 compared to both hu-mice and double hu-mice. Human donor samples had lower relative

175 abundances of the class Bacilli and family Lactobacillaceae compared to both hu-mice and

176 double hu-mice. Human donor samples also had a higher relative abundance of the class

177 Clostridia than both hu-mice and double hu-mice samples. Significant differences found within

178 this class were the family Lachnospiraceae, genus Blautia, genus Roseburia, family

179 Ruminococcaceae, genus Ruminococcus, and species Faecalibacterium prausnitzii. Some of

180 these taxa do increase in abundance in double hu-mice as compared to hu-mice, as observed

181 with significant differences in the genus Blautia and genus Ruminococcaceae. Levels of the

182 species Akkermansia muciniphila are significantly higher in hu-mice and double hu-mice

183 samples compared to human donor samples. However, double hu-mice have significantly less

184 relative abundance of this species than hu-mice samples. 
To further determine differences in relative abundances among microbiomes from the various treatments, we used Linear discriminant analysis Effect Size (LEfSe) with a P value $<.05$ and LDA score > 2 (Supplemental File - LefSe.xlsx)[23]. Taxa with LDA scores higher than 4

188 were plotted to show significant differences between double hu-mice and human donor

189 samples and double hu-mice and hu-mice samples (Fig. 5). Human donor samples were

190 associated with higher relative abundances of several types of Clostridia including

191 Lachnospiraceae, Blautia, Coprococcus, Roseburia, Facalibacterium, and Ruminococcus

192 compared to double hu-mice, while double hu-mice samples were associated with Lactobacillus

193 and Akkermansia muciniphila (Fig. 5a). Double hu-mice were associated with higher relative

194 abundances of Bacteroides and several types of Clostridia including Blautia, Coprococcus, and

195 Ruminococcaceae, while hu-mice samples were associated with S24-7 and Mogibacteriaceae

196 (Fig. 5b). By characterizing the relative bacterial abundance in double hu-mice, we

197 demonstrated that certain taxa, like members of Bacteroides, readily engrafted while others

198 such as Clostridia were more difficult to transplant. Further, several species such as those found

199 in the phylum Verrucomicrobia were highly prevalent in hu-mice, and antibiotic treatment

200 followed by fecal transplant did not fully diminish or replace this population based on relative

201 abundances. Altogether, these results demonstrate that engraftment of human donor samples

202 significantly changed the taxonomic profile of double hu-mice and that their human-like gut

203 microbiomes were statistically more similar to human donor profiles compared to hu-mice.

205 The engrafted human-like gut microbiome in double hu-mice is stable. 
To evaluate the stability of the engrafted human-like gut microbiome in double hu-mice

207 after fecal transplant, the proportion of shared amplicon sequence variants (ASVs) with the

208 human donor sample was calculated (Fig. 6). After engraftment, double hu-mice had increased

209 proportions of shared ASVs with their respective human donor samples, and those proportions

210 remained higher than pre-treatment and control levels for the duration of the study. The first

211 cohort of double hu-mice engrafted with human donor 65 had an average shared ASV

212 proportion of $13.70 \%$ after transplant, while the pre-treatment samples had $1.06 \%$ and control

213 samples levels had 1.42\% (Fig. 6a). This increased proportion of shared ASVs was maintained

214 for the duration of the study, up to 14 weeks post-transplant. A second cohort of double hu-

215 mice engrafted with human donor 65 was created and a similar increase in the proportion of

216 shared ASVs was observed (Fig. 6b). The average proportion of shared ASVs was $17.65 \%$ post-

217 transplant, while the pre-treatment samples had $2.60 \%$ and the control samples had $0.96 \%$.

218 This increased proportion of shared ASVs was maintained for the duration of the study, up to

21914.5 weeks post-transplant. Double hu-mice transplanted with the mixture of all three human

220 donors had an average shared ASV proportion of $10.91 \%$ after transplant, while the pre-

221 treatment samples had $0.31 \%$ and the control samples had $0.61 \%$ (Fig. $6 \mathrm{~d}$ ).

222 The proportion of shared ASVs was then calculated for cohorts of double hu-mice only

223 treated with 7 days of antibiotics prior to fecal transplant. Double hu-mice engrafted with

224 human donor 74 had an average shared ASV proportion of $11.85 \%$ after transplant, while the

225 pre-treatment samples had $1.02 \%$ and the control samples had $0.86 \%$ (Fig. 6c). Double hu-mice

226 transplanted with human donor 82 had an average shared ASV proportion of $10.56 \%$ after 
227 transplant, while the pre-treatment samples had $1.33 \%$ and the control samples had $0.95 \%$ (Fig.

$2286 c)$

229 To further evaluate the stability of the engrafted human-like microbiome after

230 transplant into double hu-mice, the contributions of the human donor and pre-treatment

231 sample to the post-transplant samples were determined using SourceTracker (Fig. 7)[24]. The

232 first cohort of mice transplanted with human donor 65 had an average donor contribution

233 percentage of $18.76 \%$ after transplant, while the pre-treatment samples had $0.00 \%$ and the

234 control samples had $0.05 \%$ (Fig. 7a). At the final time point collected at 14 WPT, the donor

235 contribution was consistent at $18.10 \%$. The second cohort of mice transplanted with human

236 donor 65 had an average donor contribution percentage of $29.01 \%$ after transplant, while the

237 pre-treatment and controls samples had no donor contribution (Fig. 7b). The donor

238 contribution percentage was $34.10 \%$ at 14.5 WPT, thus demonstrating the stable engraftment

239 of donor bacteria. Double hu-mice engrafted with donor 74 had an average donor contribution

240 percentage of $12.71 \%$ after transplant, while the pre-treatment samples had $0.00 \%$ and the

241 control samples had $0.00 \%$ (Fig. 7c). At the final time point collected at 6 WPT, the average

242 donor contribution was $18.75 \%$.

243 The SourceTracker algorithm was unable to clearly distinguish donor 82 contributions as

244 both pre-treatment and control samples were assigned high human donor contribution

245 percentages. Mice transplanted with human donor 82 had an average donor contribution

246 percentage of $10.18 \%$ after transplant, while the pre-treatment samples had $11.95 \%$ and the

247 control samples had 20.62\% (Fig. 7c). SourceTracker also assigned very high donor contribution

248 percentages to the pre-treatment and control samples in the study of double hu-mice 
249 transplanted with a mixture of all three human donors. Mice transplanted with the mixture of

250 all three human donors had an average donor contribution percentage of $14.88 \%$ after

251 transplant, while the pre-treatment samples had $21.04 \%$ and the control samples had $15.89 \%$

252 (Fig. 7d).

253

The high donor contribution percentages of donor 82 to the pre-treatment and control

254 samples originated from an ASV with taxonomic assignment to Akkermansia muciniphila. This

255 ASV was highly abundant in both hu-mice and double hu-mice and was much more prevalent in

256 human donor 82 and the mixed donor sample compared to donors 65 or 74 . To get a more

257 accurate account of the stability of the human-like gut microbiome in post-transplant samples,

258 we removed this ASV that was resulting in false positive donor contributions and once again

259 used SourceTracker (Supplemental Figure - SourceTracker_82_Mix.pdf). After the removal of

260 the ASV, the double hu-mice engrafted with human donor 82 had an average donor

261 contribution percentage of $12.64 \%$ after transplant and double hu-mice engrafted with the

262 mixture of all three human donors had an average donor contribution percentage of $18.11 \%$

263 after transplant, while all pre-treatment control samples were at $0.00 \%$. Using both a

264 percentage of shared ASVs and SourceTracker, we have demonstrated that double hu-mice had

265 a stable human-like gut microbiome for the duration of the study, up to 14.5 weeks post-

266 transplant.

267

268 Double hu-mice have increased human-like predicted metagenome functional content. In addition to evaluating microbiome classification, we also sought to assess the

270 functional capacity of the microbiomes in double hu-mice. PICRUSt was used to predict the 
271 metagenome functional content from the 16S rRNA data after ASV inference [25], and the

272 predicted KO features were graphed using both NMDS and PCoA plots (Fig. 8). Many of the

273 double hu-mice samples clustered closer to the human donor samples than the hu-mice

274 samples (Fig. 8a \& 8b). When color-coded by donor and cohort, the microbiomes that clustered

275 closest to the human donor samples belonged to mice from the second cohort of double hu-

276 mice generated by engrafting bacteria from human donor 65 (Fig 8c \& 8d). Similarly, several

277 samples from the other double hu-mice cohorts also separated themselves from the hu-mice

278 cluster and were closer to the human donor samples. (Fig. 8c \& 8d).

279

We next tested for differences of each predicted KO feature among the three different

280 groups of mice (Supplemental File - KO_Significant_Differences.xlsx). In total, there were 4,513

281 non-zero predicted KO features. Using Kruskal-Wallis testing and an FDR adjusted p-value of <

2820.05 , we found $35.54 \%(1,604 / 4,513)$ significantly different predicted KO features between hu-

283 mice and human donor samples. There were $39.09 \%(1,764 / 4,513)$ significantly different

284 predicted KO features between double hu-mice and hu-mice samples. However, when we

285 compared double hu-mice and the human donor samples, there were only $1.35 \%(61 / 4,513)$

286 significantly different predicted KO features. To clarify what functional aspects were missing

287 from the double hu-mice gut microbiomes, we determined the predicted ASV contribution for

288 each of the 61 significantly different KO features between the double hu-mice and human

289 donor samples (Supplemental File - KO_Metagenome_Contribution.xlsx). This analysis provided

290 insight into which functionally significant bacteria were not successfully engrafted from the

291 human donor samples to the double hu-mice. A total of 95 ASVs with 73 unique taxonomic

292 assignments were found to contribute to the 61 significantly different KO features 
293 (Supplemental Figure - KO_Contributions.pdf). Family level taxa with the highest levels of

294 contribution included Actinomycetaceae (10.46\%), Bifidobacteriaceae (8.76\%),

295 Streptococcaceae (20.74\%), Lachnospiraceae (23.95\%), and Peptococcaceae (8.66\%).

296 Bifidobacterium adolescentis was the highest contributing species (7.85\%), and the highest

297 contributing ASV (13.01\%) had the taxonomic assignment of an unclassified Streptococcus

298 species. Collectively, these analyses demonstrate that the predicted functional capacity of

299 double hu-mice is more similar to human donor samples compared to hu-mice.

\section{Discussion}

The goal of this study was to investigate the establishment and stability of an engrafted human gut microbiome after antibiotic pre-treatment in hu-mice. We call these mice double

304 hu-mice as they have both a functional human immune system and human bacterial gut

305 microbiomes similar to human donor samples. Our approach created highly reproducible and

306 donor-specific human-like gut microbiomes across multiple cohorts of double hu-mice. Further,

307 we showed that double hu-mice had increased measures of diversity and increased functional

308 capacity compared to hu-mice. The engrafted human-like gut microbiomes were also stable for

309 the duration of the study, up to 14.5 weeks post-transplant. Further, we demonstrated that the

310 predicted functional capacity of double hu-mice is also more similar to the human donor

311 samples than hu-mice.

One of the most significant aspects of the study was that the double hu-mice had gut

313 microbiome profiles that were unique to the human donor engrafted. Each of the 4 different

314 donor samples resulted in a distinct population of double hu-mice resembling the human 
315 donor. Double hu-mice create a huge opportunity for implementing personalized medicine and

316 translational research. Potential applications for double hu-mice include testing for

317 personalized responses of patient microbiomes to drug regimens, therapies, or dietary

318 interventions. These mice could also be used to identify mechanisms underlying observations

319 from human studies establishing a connection between the gut microbiome and disease.

320 In this study, we created a cohort of double hu-mice by engrafting a mixture of all three

321 human donors to create an 'un-biased' human gut microbiome profile. Further study is needed

322 to determine whether a mixed sample is beneficial in creating an un-biased human-like profile

323 or if mixing samples creates an un-natural or unstable community of gut bacteria after

324 engraftment. It may be advantageous to use a mixture of fecal samples derived from a large

325 population to determine the broad impact of different treatments or diets to the human gut

326 microbiome. This un-biased mix of a large population of donors could be used to add additional

327 information to double hu-mice generated with individual donor profiles.

328 We found that double hu-mice had increased measures of alpha diversity compared to

329 hu-mice. Several studies have highlighted the importance of microbiome diversity within the

330 gut and have linked low gut microbiome diversity with several disease conditions[26, 27]. While

331 not all low diversity conditions are detrimental, specifically when there is enrichment of

332 potentially beneficial bacteria through prebiotic or probiotic treatment, the low pre-existing

333 diversity found within the hu-mice was far below the levels observed in our human donor

334 samples. After engraftment, the double hu-mice had increased levels of alpha diversity and

335 maybe more importantly, had increased predicted functional capacities. This increased diversity 
336 may also offer a more realistic gut environment as it allows for diverse reciprocal interactions

337 with the engrafted human immune system.

We also found that the engrafted human-like microbiome was very stable in our model

339 for the length of the study, up to 14.5 weeks after transplant. We used several methods to

340 determine the engraftment level and stability of the gut microbiome after transplant and found

341 no reversion to the pre-existing murine profile. This stability allows study of the role of the gut

342 microbiome in many human diseases such as HIV-1 and cancer. One outstanding question is

343 whether the unique presence of human immune cells plays a role in stabilizing or enhancing

344 engraftment of the human-like gut microbiome in our model compared to other non-

345 humanized mouse models. Our data showed no reversion to the pre-existing murine gut

346 microbiome profile, perhaps due to some enhanced stability or selection by the reconstituted

347 human immune system. Further studies are needed to determine the relationship between the

348 engrafted gut microbiome and human immune system.

Many different methods and antibiotic regimens have been used for preconditioning of

350 mice prior to fecal transplantation[28-30]. Different combinations and durations of antibiotic

351 treatments may increase the efficiency of the fecal transplant into the host[29]. While the

352 combination of Metronidazole, Ampicililn, Neomycin, and Vancomycin is widely used due to its

353 broad spectrum of bacterial targets, the best methods are still being investigated. We found

354 that the very rigorous method of gavaging antibiotics twice daily for 14 days used by Hintze et

355 al. was too invasive for our NSG hu-mice and resulted in increased mortality[31]. Providing the

356 antibiotics in the drinking water proved to be less stressful with improved health and survival of 
357 the mice. Meanwhile, we also found that 14 days was the optimum duration of antibiotic pre-

358 treatment to generate double hu-mice.

As expected, there is not a complete reconstitution of the human fecal donor profile in our double hu-mice due to several hypothesized reasons. There are major differences between

361 the human and mouse digestive tract including structure, function, and $\mathrm{pH}[19]$. Our mice are

362 not germ-free to begin with, and the broad-spectrum antibiotic treatment can only reduce the

363 prevalence of pre-existing murine gut bacteria. There were several key differences in the

364 reconstituted mice compared to the human donors. Double hu-mice had significantly lower

365 levels of several types of Clostridia including Lachnospiraceae, Blautia, Coprococcus, Roseburia,

366 Faecalibacterium, and Ruminococcus compared to human donor samples. Many of these

367 bacteria are well documented to be difficult to reconstitute within germ-free and SPF mouse

368 models[20, 31, 32]. Similar to fecal transplants in humans designed to treat $C$. difficile

369 infections, the engrafted human-like gut microbiome in our double hu-mice is the result of a

370 combination of host, donor, and environmental bacteria[33]. Despite these previously known

371 limitations, our double hu-mice model reproducibly results in a donor specific, stable, human-

372 like gut microbiome in the presence of a human immune system.

373 Germ-free animals are the gold standard for studying the gut microbiome. Using germ-

374 free mice to study the impact of the gut microbiome has been well-documented[1, 20]. Germ-

375 free animal models may allow for a more complete reconstitution of a human-like gut

376 microbiome following fecal transplant, however these models often do not have human

377 immune system. Studying human immune reconstitution in hu-mice and pathogenesis of

378 human specific diseases in a gnotobiotic environment could reveal important clues about the 
379 role of the gut microbiome. Nevertheless, many important mouse strains are not commercially

380 available as germ-free, including NSG mice. Several studies have also shown that gnotobiotic

381 mice may have long-lasting immune deficiencies, even after gut microbiome reconstitution[34-

382 36]. Further, working with germ-free animals requires gnotobiotic facilities and equipment that

383 is expensive and has limited availability. Lundberg et al., and Kennedy et al., nicely review the

384 advantages and disadvantages of using antibiotic-treated versus germ-free rodents for

385 microbiota transplantation studies [37, 38].

Our double hu-mice have the advantage of requiring only SPF housing conditions, which

387 are widely available and less expensive compared to germ-free facilities. It also does not

388 perturb the complex surgical procedures in generating BLT hu-mice because there is no need

389 for a completely germ-free environment. Our NSG mice are housed and bred under SPF

390 conditions and the diversity of their murine gut microbiota is low. Their immunodeficiency may

391 contribute to their pre-existing low diversity gut microbiome status before human fecal

392 material transplant. In a study by Zhou et al., NSG and C57BL6/J mice whose native microbiota

393 were depleted by antibiotics followed by FMT had no significant differences in diversity but did

394 observe significant differences in which species colonized[39]. A study done by Ericsson et al.

395 showed that it is easier to transfer high diversity fecal donor samples into low diversity

396 recipients, which could help to explain the success of engraftment and stability in our

397 model[30]. Many questions remain as to the best antibiotic preconditioning regimen, the timing

398 of fecal transplants, the total number of fecal transplants, the route of administration (oral

399 versus rectal), the use of antacids, and preconditioning with osmotic laxatives such as 
400 polyethylene glycol, diet, and housing. Methods to optimize murine bacterial depletion along

401 with reconstitution and stability of human specific bacteria are currently being explored.

402

\section{Conclusion}

Here, we describe successful and stable transplantation of human fecal microbiomes into immunodeficient NSG mice surgically engrafted with a functional human immune system

406 to create double hu-mice with human donor-specific human gut microbiomes. Double hu-mice

407 will be beneficial to many applications of personalized medicine to test the impact of the

408 human gut microbiome on human health and disease in the presence of a human immune

409 system.

\section{Methods}

\section{Generation of humanized BLT mice}

413 All methods described here were conducted as we previously reported in accordance

414 with Institutional Animal Care and Research Committee (IACUC)-approved protocols at the

415 University of Nebraska-Lincoln (UNL)[22, 40-42]. The IACUC at the University of Nebraska-

416 Lincoln (UNL) has approved two protocols related to generating and using humanized BLT (hu-

417 BLT) mice, including Double Hu-Mice. Additionally, the Scientific Research Oversight Committee

418 (SROC) at UNL has also approved the use of human embryonic stem cells and fetal tissues,

419 which are procured from the Advanced Bioscience Resources for humanized mice studies

420 (SROC\# 2016-1-002). 
422 number 005557; (Jackson Laboratory) were housed and maintained in individual microisolator

423 cages in a rack system capable of managing air exchange with prefilters and HEPA filters. Room

424 temperature, humidity, and pressure were controlled, and air was also filtered. Mice were fed

425 irradiated Teklad global 14\% protein rodent chow (Teklad 2914) and were given autoclaved

426 acidified drinking water. The second cohort of double hu-mice engrafted with fecal material

427 from Donor 65 were supplemented with a high calorie gel (DietGel Boost). On the day of

428 surgery, mice received whole-body irradiation at the dose of $12 \mathrm{cGy} / \mathrm{gram}$ of body weight with

429 the RS200 X-ray irradiator (RAD Source Technologies, Inc., GA) and were then implanted with

430 one piece of human fetal thymic tissue fragment sandwiched between two pieces of human

431 fetal liver tissue fragments within the murine left renal capsule. Within 6 hours of surgery, mice

432 were injected via the tail vein with $1.5 \times 10^{5}$ to $5 \times 10^{5} \mathrm{CD} 34^{+}$hematopoietic stem cells isolated

433 from human fetal liver tissues. Human fetal liver and thymus tissues were procured from

434 Advanced Bioscience Resources (Alameda, CA). After 9 to 12 weeks, human immune cell

435 reconstitution in peripheral blood was measured by a fluorescence-activated cell sorter (FACS)

436 Aria Il flow cytometer (BD Biosciences, San Jose, CA) using antibodies against mCD45-APC,

437 hCD45-FITC, hCD3-PE, hCD19-PE/Cy5, hCD4-Alexa 700, and hCD8-APC-Cy7 (catalog numbers

438 103111, 304006, 300408, 302209, 300526, and 301016, respectively; BioLegend, San Diego,

439 CA). Raw data were analyzed with FlowJo (version 10.0; FlowJo LLC, Ashland, OR). All mice used

440 in this study had high levels of human immune cell reconstitution with an average of $85 \%$

441 hCD45+ cells in peripheral blood 10 weeks post-surgery. The mice were randomly assigned into

442 experimental groups with similar immune reconstitution levels. 
Antibiotic treatment

A broad-spectrum antibiotic cocktail was prepared fresh daily consisting of

446 Metronidazole (1 g/L), Neomycin (1 g/L), Vancomycin (0.5 g/L), and Ampicillin (1 g/L). The

447 antibiotic cocktail was given to the mice ad libitum in the drinking water along with grape

448 flavored Kool-Aid to improve palatability. Control group mice were given only grape flavored

449 Kool-Aid in the drinking water. During antibiotic treatment, cages were changed daily to limit

450 re-inoculation of pre-existing bacteria to the mice due to their coprophagic behavior.

451 Antibiotics were given for 14 days for double hu-mice reconstituted with Donor 65 and Donor

452 Mix and for 7 days in double hu-mice reconstituted with Donor 74 and Donor 82 . Mice in the

453 Pilot Study were given antibiotics via oral gavage. Mice were first given three days of anti-fungal

454 Amphotericin B treatment $(1 \mathrm{mg} / \mathrm{kg})$ twice daily via oral gavage. Mice were then given the

455 antibiotic cocktail along with Amphotericin B via twice daily via oral gavage. After 4 days of

456 treatment, the Amphotericin B was stopped due to toxicity concerns and after 10 days of

457 treatment oral gavage was reduced to once daily. Post-antibiotic treatment, mice were given

458 autoclaved non-acidified deionized drinking water.

459 During the first few days of antibiotic treatment, the mice lost a considerable amount of

460 body weight (10-20\%). The weight loss plateaued at 3-4 days and remained steady for the

461 remainder of antibiotic treatment. Body weight was carefully monitored during this time and If

462 needed, mice were treated with Intraperitoneal (IP) injections of Ringer's solution to mitigate

463 any effects of dehydration. After fecal transplant, the mice began to regain weight and returned

464 to their pre-existing weight within 2 weeks post-transplant. During antibiotic treatment, there 
465 was a large reduction in spleen size and a large increase in cecum size compared to controls.

466 This is similar to the morphology observed in germ-free mice, providing further evidence for the

467 efficacy of the antibiotic regimen[35].

468

Donor samples and Fecal transplant

At 24 and 48 hours after the completion of antibiotic pre-treatment, mice were given

471200 ul of human fecal material via oral gavage. OpenBiome supplied 3 FMT Upper Delivery

472 Microbiota Preparations from 3 different healthy human donors (Donor 65, Donor 74, Donor

473 82). Samples were thawed once before fecal transplant to aliquot the samples within an

474 anaerobic chamber. During this step, an equal portion of each of the samples were mixed

475 together to create an unbiased human donor sample (Donor Mix). 16S rRNA sequencing data

476 on the three donors was also supplied by OpenBiome (Supplementary data).

478 Mouse fecal collection and DNA extraction

480 fresh fecal samples were produced. Fecal samples were stored in $1.5 \mathrm{ml}$ Eppendorf tubes at -80

$481{ }^{\circ} \mathrm{C}$ until DNA extraction. DNA was extracted from the fecal samples using the

482 phenol:chloroform:isoamyl alcohol with bead beating method described previously [43]. Briefly,

483 fecal samples were washed three times with $1 \mathrm{ml}$ PBS buffer (pH 7). After the addition of 750 ul

484 of lysis buffer, samples were transferred to tubes containing $300 \mathrm{mg}$ of autoclaved $0.1 \mathrm{~mm}$

485 zirconia/silica beads (Biospec). 85 ul of 10\% SDS solution and 40 ul of Proteinase K (15mg/ml,

486 MC500B Promega) were added and samples were incubated for 30 minutes at $60^{\circ} \mathrm{C} .500 \mathrm{ul}$ of 
487 Phenol:Chloroform:Isoamyl alcohol (25:24:1) was added and then samples were vortexed.

488 Samples were then put into a bead beater (Mini-beadbeater 16 Biospec) for 2 minutes to

489 physically lyse the cells. The upper phase of the sample was collected and an additional 500ul of

490 Phenol:Chloroform:Isoamyl alcohol (25:24:1) was added. After samples were vortexed and spun

491 down, the DNA in the upper phase was further purified twice with 500 ul of

492 Phenol:Chloroform:Isoamyl alcohol (25:24:1). and was then precipitated with 100\% Ethanol (2.5

$493 \times$ volume of sample) and $3 \mathrm{M}$ Sodium acetate $\left(.1 \times\right.$ volume of sample) overnight at $-20^{\circ} \mathrm{C}$.

494 Samples are then centrifuged and dried at room temperature. DNA was resuspended in $100 \mathrm{ul}$

495 of Tris-Buffer $(10 \mathrm{mM}, \mathrm{pH} 8)$ and stored at $-20^{\circ} \mathrm{C}$. DNA samples were quality checked by

496 nanodrop (ND-1000 Nanodrop) .

497

$498 \quad 16 S$ rRNA gene sequencing

499 16S rRNA gene sequencing was performed at the University of Nebraska Medical Center

500 Genomics Core Facility using xxx (detailed Illumina instrument here). DNA normalization and

501 library prep were performed followed by V3-V4 16S rRNA amplicon gene sequencing using a

502 MiSeqV2 (Illumina) The following primer sequences were used: (Primer sequences: Forward

503 Primer $=5^{\prime}$

504 TCGTCGGCAGCGTCAGATGTGTATAAGAGACAGCCTACGGGNGGCWGCAG 16S Amplicon PCR

505 Reverse Primer $=5^{\prime}$

506 GTCTCGTGGGCTCGgAGATGTGTATAAGAGACAGGACTACHVGGGTATCTAATCC 
507 Illumina overhangs: Forward overhang: 5'

508 TCGTCGGCAGCGTCAGATGTGTATAAGAGACAG-[locusspecific sequence] Reverse overhang: 5’

509 GTCTCGTGGGCTCGGAGATGTGTATAAGAGACAG-[locusspecific sequence]).

511 Generation of the amplicon sequence variant table and data analysis

512 Illumina-sequenced paired-end fastq files were demultiplexed by sample and barcodes

513 were removed by the sequencing facility. The University of Nebraska Holland Computer Center

514 Crane cluster was used to run the DADA2 v1.8 $\mathrm{R}$ package in order to generate an amplicon

515 sequence variant (ASV) table[44]. An example of the script used to generate the ASV table is

516 provided in the supplementary materials. The DADA2 pipeline was performed as follows,

517 sequences were filtered and trimmed during which any remaining primers, adapters, or linkers

518 were also removed. The sequencing error rates were estimated using a random subset of the

519 data. Dereplication of the data combined all identical sequencing reads into unique sequences

520 with a corresponding abundance. The core sample inference algorithm was then applied to the

521 dereplicated data. The forward and reverse reads were then joined to create the full denoised

522 sequences and an initial ASV table was generated. Any sequences outside the expected length

523 for the V3-V4 amplicon were then filtered from the table. Chimeric sequences were then

524 removed and a final ASV table was generated. Taxonomy was assigned using the Greengenes

52513.8 database and RDP Classifier with a minimal confidence score of $0.80[45,46]$.

527 Data analysis

528 Analysis was performed using R package mctoolsr (https://github.com/leffj/mctoolsr/). 
529 and samples were rarified to 13,000 ASVs for downstream analysis. Additional testing of

530 differences between groups was performed using LEfSe[23]. SourceTracker was used to

531 evaluate the stability of the transferred donor microbiome in the double hu-mice[24].

532 GraphPad Prism 5 were used to create some figures. DADA2 generated ASVs were used to

533 predict the functional metagenome capacity using PICRUSt[25] via the following pipeline

534 (https://github.com/vmaffei/dada2_to_picrust).

\section{Data availability}

537 The datasets generated during the current study are available in the NCBI SRA repository,

538 [https://www.ncbi.nlm.nih.gov/bioproject/PRJNA507247].

\section{$\underline{\text { References }}$}

543 1. Turnbaugh PJ, Ley RE, Mahowald MA, Magrini V, Mardis ER, Gordon JI: An obesityassociated gut microbiome with increased capacity for energy harvest. Nature 2006, 444:1027-1031.

2. Gopalakrishnan V, Spencer CN, Nezi L, Reuben A, Andrews MC, Karpinets TV, Prieto PA, Vicente D, Hoffman K, Wei SC, et al: Gut microbiome modulates response to anti-PD-1 immunotherapy in melanoma patients. Science 2018, 359:97-103.

3. Routy B, Le Chatelier E, Derosa L, Duong CPM, Alou MT, Daillere R, Fluckiger A, Messaoudene M, Rauber C, Roberti MP, et al: Gut microbiome influences efficacy of PD-1-based immunotherapy against epithelial tumors. Science 2018, 359:91-+.

4. Clemente JC, Manasson J, Scher JU: The role of the gut microbiome in systemic inflammatory disease. Bmj-British Medical Journal 2018, 360.

5. Qin J, Li R, Raes J, Arumugam M, Burgdorf KS, Manichanh C, Nielsen T, Pons N, Levenez $F$, Yamada $T$, et al: $A$ human gut microbial gene catalogue established by metagenomic sequencing. Nature 2010, 464:59-65.

6. Kau AL, Ahern PP, Griffin NW, Goodman AL, Gordon JI: Human nutrition, the gut microbiome and the immune system. Nature 2011, 474:327-336. 
559 7. Hooper LV, Littman DR, Macpherson AJ: Interactions Between the Microbiota and the $560 \quad$ Immune System. Science 2012, 336:1268-1273.

561 8. Maynard CL, Elson CO, Hatton RD, Weaver CT: Reciprocal interactions of the intestinal microbiota and immune system. Nature 2012, 489:231-241.

563

9. Simpson-Abelson MR, Sonnenberg GF, Takita H, Yokota SJ, Conway TF, Kelleher RJ, Shultz LD, Barcos M, Bankert RB: Long-term engraftment and expansion of tumorderived memory $T$ cells following the implantation of non-disrupted pieces of human lung tumor into NOD-scid IL2R gamma(null) mice. Journal of Immunology 2008, 180:7009-7018.

10. Bankert RB, Balu-lyer SV, Odunsi K, Shultz LD, Kelleher RJ, Barnas JL, Simpson-Abelson M, Parsons R, Yokota SJ: Humanized Mouse Model of Ovarian Cancer Recapitulates Patient Solid Tumor Progression, Ascites Formation, and Metastasis. Plos One 2011, 6.

11. Vudattu NK, Waldron-Lynch F, Truman LA, Deng SY, Preston-Hurlburt P, Torres R, Raycroft MT, Mamula MJ, Herold KC: Humanized Mice as a Model for Aberrant Responses in Human T Cell Immunotherapy. Journal of Immunology 2014, 193:587-596.

12. Whitfield-Larry F, Young EF, Talmage G, Fudge E, Azam A, Patel S, Largay J, Byrd W, Buse J, Calikoglu AS, et al: HLA-A2 Matched Peripheral Blood Mononuclear Cells From Type 1 Diabetic Patients, but Not Nondiabetic Donors, Transfer Insulitis to NOD-scid/gamma c(null)/HLA-A2 Transgenic Mice Concurrent With the Expansion of Islet-Specific CD8(+) T cells. Diabetes 2011, 60:1726-1733.

13. Yi GH, Xu XQ, Abraham S, Petersen S, Guo H, Ortega N, Shankar P, Manjunath N: A DNA Vaccine Protects Human Immune Cells against Zika Virus Infection in Humanized Mice. Ebiomedicine 2017, 25:87-94.

14. Stary G, Olive A, Radovic-Moreno AF, Gondek D, Alvarez D, Basto PA, Perro M, Vrbanac VD, Tager AM, Shi JJ, et al: A mucosal vaccine against Chlamydia trachomatis generates two waves of protective memory T cells. Science 2015, 348.

15. Sun ZF, Denton PW, Estes JD, Othieno FA, Wei BL, Wege AK, Melkus MW, PadgettThomas A, Zupancic M, Haase AT, Garcia JV: Intrarectal transmission, systemic infection, and CD4(+) T cell depletion in humanized mice infected with HIV-1. Journal of Experimental Medicine 2007, 204:705-714.

16. Wang LX, Kang GB, Kumar P, Lu WX, Li Y, Zhou Y, Li QS, Wood C: Humanized-BLT mouse model of Kaposi's sarcoma-associated herpesvirus infection. Proceedings of the National Academy of Sciences of the United States of America 2014, 111:3146-3151.

17. Ernst W: Humanized mice in infectious diseases. Comparative Immunology Microbiology and Infectious Diseases 2016, 49:29-38.

18. Xiao L, Feng Q, Liang SS, Sonne SB, Xia ZK, Qiu XM, Li XP, Long H, Zhang JF, Zhang DY, et al: A catalog of the mouse gut metagenome. Nature Biotechnology 2015, 33:1103-+.

19. Nguyen TLA, Vieira-Silva S, Liston A, Raes J: How informative is the mouse for human gut microbiota research? Disease Models \& Mechanisms 2015, 8:1-16.

20. Turnbaugh PJ, Ridaura VK, Faith JJ, Rey FE, Knight R, Gordon JI: The Effect of Diet on the Human Gut Microbiome: A Metagenomic Analysis in Humanized Gnotobiotic Mice. Science Translational Medicine 2009, 1.

601 21. Hazenberg MP, Bakker M, Verschoor-Burggraaf A: Effects of the human intestinal flora on germ-free mice. J Appl Bacteriol 1981, 50:95-106. 
22. Daharsh L Z, Ramer-Tait A, Li Q: A Double Humanized BLT-mice Model Featuring a Stable Human-Like Gut Microbiome and Human Immune System. Jove-Journal of Visualized Experiments 2019.

23. Segata N, Izard J, Waldron L, Gevers D, Miropolsky L, Garrett WS, Huttenhower C: Metagenomic biomarker discovery and explanation. Genome Biol 2011, 12:R60.

24. Knights D, Kuczynski J, Charlson ES, Zaneveld J, Mozer MC, Collman RG, Bushman FD, Knight R, Kelley ST: Bayesian community-wide culture-independent microbial source tracking. Nat Methods 2011, 8:761-763.

25. Langille MG, Zaneveld J, Caporaso JG, McDonald D, Knights D, Reyes JA, Clemente JC, Burkepile DE, Vega Thurber RL, Knight $R$, et al: Predictive functional profiling of microbial communities using 16S rRNA marker gene sequences. Nat Biotechnol 2013, 31:814-821.

26. Willing BP, Dicksved J, Halfvarson J, Andersson AF, Lucio M, Zheng Z, Jarnerot G, Tysk C, Jansson JK, Engstrand L: A Pyrosequencing Study in Twins Shows That Gastrointestinal Microbial Profiles Vary With Inflammatory Bowel Disease Phenotypes. Gastroenterology 2010, 139:1844-U1105.

27. Chang JY, Antonopoulos DA, Kalra A, Tonelli A, Khalife WT, Schmidt TM, Young VB: Decreased diversity of the fecal microbiome in recurrent Clostridium difficileassociated diarrhea. Journal of Infectious Diseases 2008, 197:435-438.

28. Reikvam DH, Erofeev A, Sandvik A, Grcic V, Jahnsen FL, Gaustad P, McCoy KD, Macpherson AJ, Meza-Zepeda LA, Johansen FE: Depletion of murine intestinal microbiota: effects on gut mucosa and epithelial gene expression. PLOS One 2011, 6:e17996.

29. Staley C, Kaiser T, Beura LK, Hamilton MJ, Weingarden AR, Bobr A, Kang J, Masopust D, Sadowsky MJ, Khoruts A: Stable engraftment of human microbiota into mice with a single oral gavage following antibiotic conditioning. Microbiome 2017, 5.

30. Ericsson AC, Personett AR, Turner G, Dorfmeyer RA, Franklin CL: Variable Colonization after Reciprocal Fecal Microbiota Transfer between Mice with Low and High Richness Microbiota. Frontiers in Microbiology 2017, 8:1-13.

31. Hintze KJ, Cox JE, Rompato G, Benninghoff AD, Ward RE, Broadbent J, Lefevre M: Broad scope method for creating humanized animal models for animal health and disease research through antibiotic treatment and human fecal transfer. Gut Microbes 2014, 5:183-191.

32. Wos-Oxley M, Bleich A, Oxley AP, Kahl S, Janus LM, Smoczek A, Nahrstedt H, Pils MC, Taudien $S$, Platzer $M$, et al: Comparative evaluation of establishing a human gut microbial community within rodent models. Gut Microbes 2012, 3:234-249.

33. Smillie CS, Smith MB, Friedman J, Cordero OX, David LA, Alm EJ: Ecology drives a global network of gene exchange connecting the human microbiome. Nature 2011, 480:241244.

34. Chung HC, Pamp SJ, Hill JA, Surana NK, Edelman SM, Troy EB, Reading NC, Villablanca EJ, Wang S, Mora JR, et al: Gut Immune Maturation Depends on Colonization with a HostSpecific Microbiota. Cell 2012, 149:1578-1593. 
35. Smith K, McCoy KD, Macpherson AJ: Use of axenic animals in studying the adaptation of mammals to their commensal intestinal microbiota. Seminars in Immunology 2007, 19:59-69.

36. Hansen CHF, Nielsen DS, Kverka M, Zakostelska Z, Klimesova K, Hudcovic T, TlaskalovaHogenova H, Hansen AK: Patterns of Early Gut Colonization Shape Future Immune Responses of the Host. Plos One 2012, 7.

37. Lundberg R, Toft MF, August B, Hansen AK, Hansen CHF: Antibiotic-treated versus germ-free rodents for microbiota transplantation studies. Gut Microbes 2016, 7:68-74.

38. Kennedy EA, King KY, Baldridge MT: Mouse Microbiota Models: Comparing Germ-Free Mice and Antibiotics Treatment as Tools for Modifying Gut Bacteria. Frontiers in Physiology 2018, 9.

39. Zhou W, Chow KH, Fleming E, Oh J: Selective colonization ability of human fecal microbes in different mouse gut environments. ISME J 2018.

40. Li QS, Tso FY, Kang GB, Lu WX, Li Y, Fan WJ, Yuan Z, Destache CJ, Wood C: Early Initiation of Antiretroviral Therapy Can Functionally Control Productive HIV-1 Infection in Humanized-BLT Mice. Jaids-Journal of Acquired Immune Deficiency Syndromes 2015, 69:519-527.

41. Destache CJ, Mandal S, Yuan Z, Kang G, Date AA, Lu W, Shibata A, Pham R, Bruck P, Rezich M, et al: Topical Tenofovir Disoproxil Fumarate Nanoparticles Prevent HIV-1 Vaginal Transmission in a Humanized Mouse Model. Antimicrob Agents Chemother 2016, 60:3633-3639.

42. Yuan Z, Kang G, Ma F, Lu W, Fan W, Fennessey CM, Keele BF, Li Q: Recapitulating CrossSpecies Transmission of Simian Immunodeficiency Virus SIVcpz to Humans by Using Humanized BLT Mice. J Virol 2016, 90:7728-7739.

43. Martinez I, Wallace G, Zhang C, Legge R, Benson AK, Carr TP, Moriyama EN, Walter J: Diet-induced metabolic improvements in a hamster model of hypercholesterolemia are strongly linked to alterations of the gut microbiota. Appl Environ Microbiol 2009, 75:4175-4184.

44. Callahan BJ, McMurdie PJ, Rosen MJ, Han AW, Johnson AJ, Holmes SP: DADA2: Highresolution sample inference from Illumina amplicon data. Nat Methods 2016, 13:581583.

45. Wang Q, Garrity GM, Tiedje JM, Cole JR: Naive Bayesian classifier for rapid assignment of rRNA sequences into the new bacterial taxonomy. Applied and Environmental Microbiology 2007, 73:5261-5267.

46. Caporaso JG, Kuczynski J, Stombaugh J, Bittinger K, Bushman FD, Costello EK, Fierer N, Pena AG, Goodrich JK, Gordon JI, et al: QIIME allows analysis of high-throughput community sequencing data. Nature Methods 2010, 7:335-336.

\section{Acknowledgements}


685 We would like to thank Yanmin Wan, Guobin Kang, and Pallabi Kundu for their assistance in

686 generating BLT hu-mice. We would like to acknowledge the UNMC Genomics Core Facility who

687 receives partial support from the Nebraska Research Network In Functional Genomics NE-INBRE

688 P20GM103427-14, The Molecular Biology of Neurosensory Systems CoBRE P30GM110768, The

689 Fred \& Pamela Buffett Cancer Center - P30CA036727, The Center for Root and Rhizobiome

690 Innovation (CRRI) 36-5150-2085-20, and the Nebraska Research Initiative. We would like to

691 thank University of Nebraska - Lincoln Life Sciences Annex and their staff for their assistance.

692 This study is supported in part by the National Institutes of Health (NIH) Grants R01Al124804

693 (to Javis), R33Al122377 (Planelles), P30 MH062261-16A1 Chronic HIV Infection and Aging in

694 NeuroAIDS (CHAIN) Center (to Buch \& Fox), 1R01AI111862 and R21 Al143405 to Q Li. The

695 funders had no role in study design, data collection and analysis, preparation of the manuscript

696 or decision for publication.

697

698 Author contributions

699 LD and QL designed the experiments and wrote the manuscript. LD performed experiments and

700 analyzed the data. ART provided input on experimental design and manuscript preparation.

701

702 Competing interests

703 The author(s) declare no competing interests.

704

705 Figure Legends 
Figure 1. Gut microbiomes of double hu-mice are distinct and more human-like compared to

707 hu-mice and feature donor specific profiles. A) Non-metric multidimensional scaling (NMDS)

708 plot displaying double hu-mice as a distinct cluster between the human donor samples and pre-

709 treatment or untreated control hu-mice. B) Principal coordinate analysis (PCoA) plot showing

710 the double hu-mice cluster with the human donor samples distinct from the pre-treatment or

711 untreated control hu-mice. C) NMDS plot displaying human donor specific profiles in the double

712 hu-mice. D) PCoA plot showing human donor specific profiles in the double hu-mice.

714 Figure 2. The gut microbiomes of double hu-mice cluster with that of human donor fecal

715 samples. Dendrogram based on Bray-Curtis distances for the gut microbiome profiles of pre-

716 treatment and untreated control hu-mice (Pre-treatment or Control Hu-Mice), double hu-mice

717 (Double Hu-Mice), antibiotic treated hu-mice (Antibiotic Treated Hu-mice), and human donor

718 fecal samples (Human Donor).

Figure 3. The gut microbiomes of double hu-mice have increased alpha diversity measures

721 compared to that of pre-treatment or untreated control hu-mice. A) Species richness, B)

722 Shannon index, and C) Simpson index. Data are shown for pre-treatment or untreated control

723 hu-mice (Hu-Mice), antibiotic treated mice (Antibiotics), double hu-mice (Double Hu-Mice), and

724 human donor fecal samples (Human Donor).

725

726 Figure 4. Comparison of relative abundance of taxa grouped by Family. The 11 most abundant

727 taxa by relative percent abundance grouped by Family are shown for pre-treatment and 
728 untreated control hu-mice (Hu-mice), double hu-mice (Double), and human fecal donor

729 samples (Donor).

731 Figure 5. Engraftment of human fecal donor bacteria in double hu-mice as shown by LEfSe. A)

732 All significant features with a linear discriminant analysis (LDA) score $>4.0$ between human

733 fecal donor samples (Donor) and double hu-mice (Double). B) All significant features with an

734 LDA score $>4.0$ between double hu-mice (Double) and pre-treatment or untreated control hu-

735 mice (Hu-Mice).

736

737 Figure 6. Engraftment and stability of a human-like gut microbiome in double hu-mice as

738 determined by shared amplicon sequence variants (ASVs). A) Proportion of shared ASVs with

739 the donor in the first cohort of double hu-mice created using fecal material from human donor

74065 (Donor 65). B) Proportion of shared ASVs with donor in the second cohort of double hu-mice

741 created using fecal material from human donor 65 (Donor 65). C) Proportion of shared ASVs

742 with donor in in double hu-mice created using fecal material from human donor 74 (Donor 74)

743 or 82 (Donor 82). D) Proportion of shared ASVs with donor in double hu-mice created using a

744 mixture of fecal material from all three human donors (Donor Mix). X-axis numbers represent

745 number of weeks post fecal transplant.

747 Figure 7. Stability of the engrafted human-like gut microbiome in double hu-mice as

748 determined by SourceTracker. A) Contributions of the human fecal donor sample and pre-

749 treatment sample in the first cohort of double hu-mice created using fecal material from 
750 human donor 65 (Donor 65). B) Contributions of the human fecal donor sample and pre-

751 treatment sample in the second cohort of double hu-mice created using fecal material from

752 human donor 65 (Donor 65). C) Contributions of the human fecal donor sample and pre-

753 treatment sample in double hu-mice created using fecal material from human donor 74 (Donor

754 74) or 82 (Donor 82). D) Contributions of the human fecal donor sample and pre-treatment

755 sample in double hu-mice created using a mixture of fecal material from all three human donor

756 (Donor Mix). X-axis numbers represent number of weeks post fecal transplant.

758 Figure 8. Double hu-mice have increased human-like predicted metagenome functional

759 content. A) NMDS plot displaying the double hu-mice as a distinct cluster between the human

760 fecal donor samples and pre-treatment or untreated control hu-mice. B) NMDS plot displaying

761 human donor specific functional profiles in double hu-mice. C) PCoA plot displaying the double

762 hu-mice as a distinct cluster between the human fecal donor samples and pre-treatment or

763 untreated control hu-mice. D) PCoA plot displaying human donor specific functional profiles in

764 double hu-mice. 
a

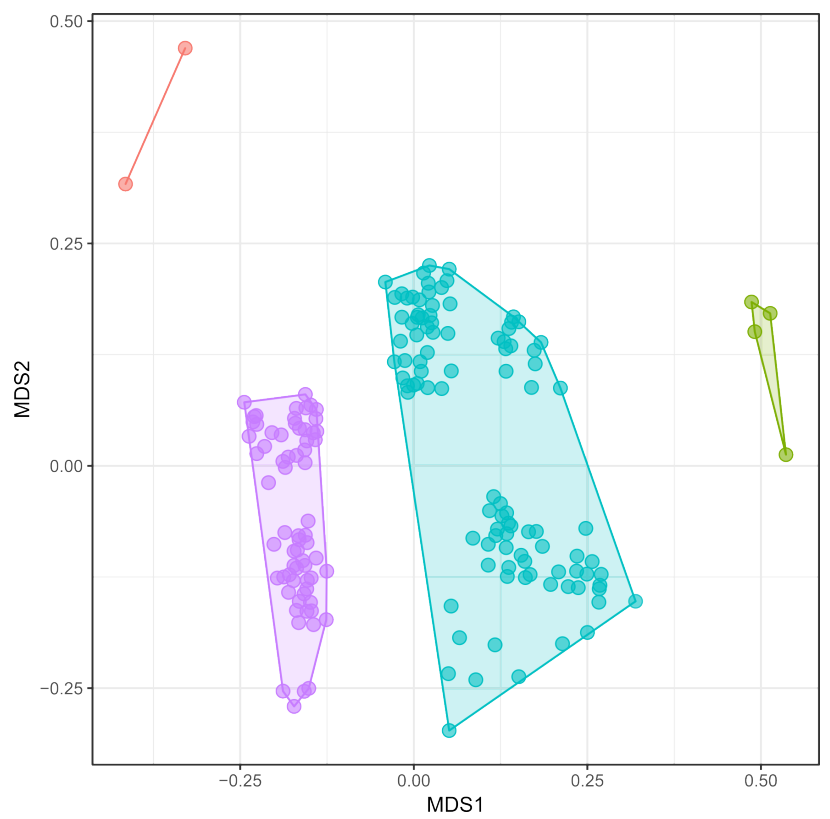

C

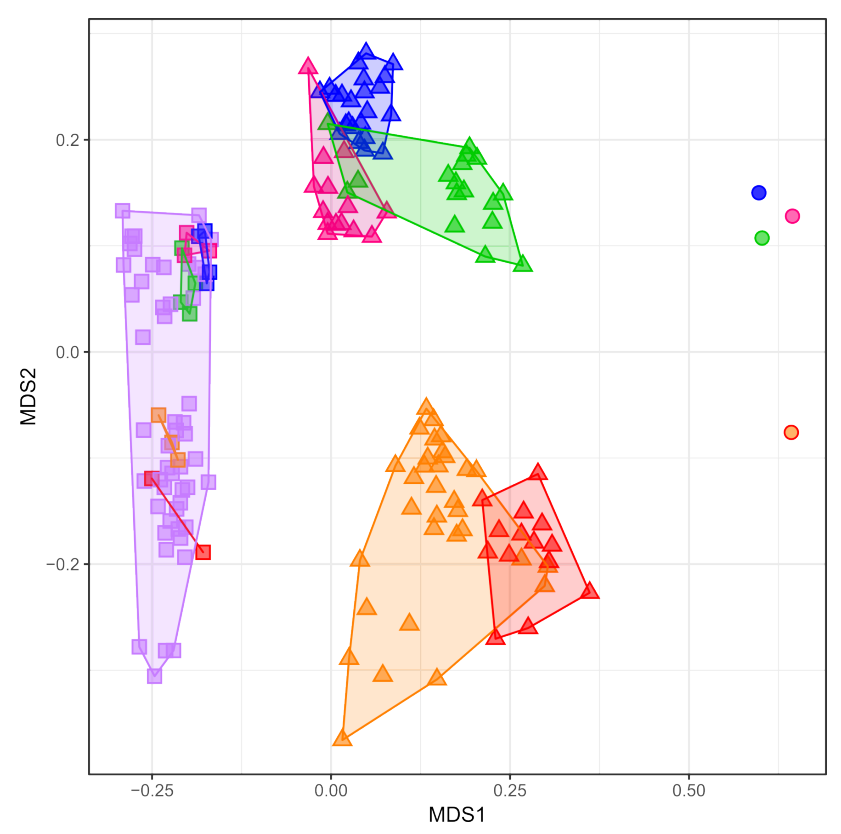

b
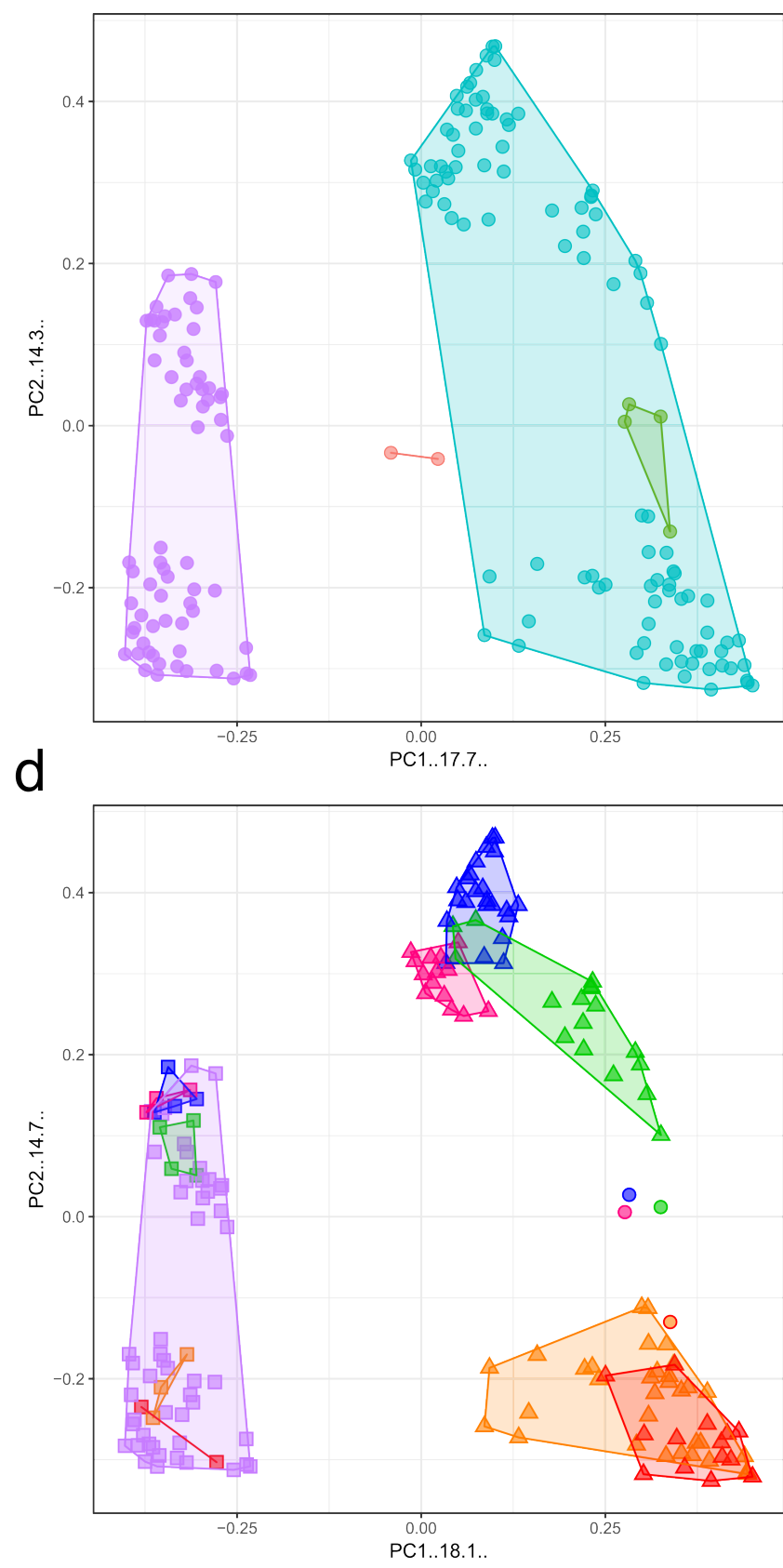

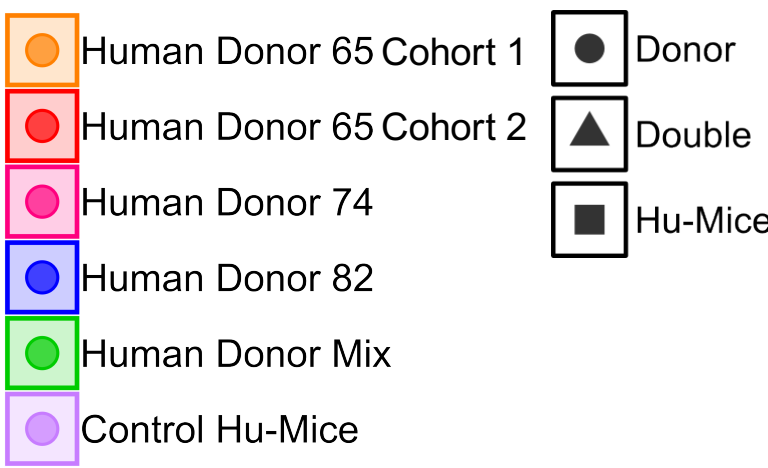




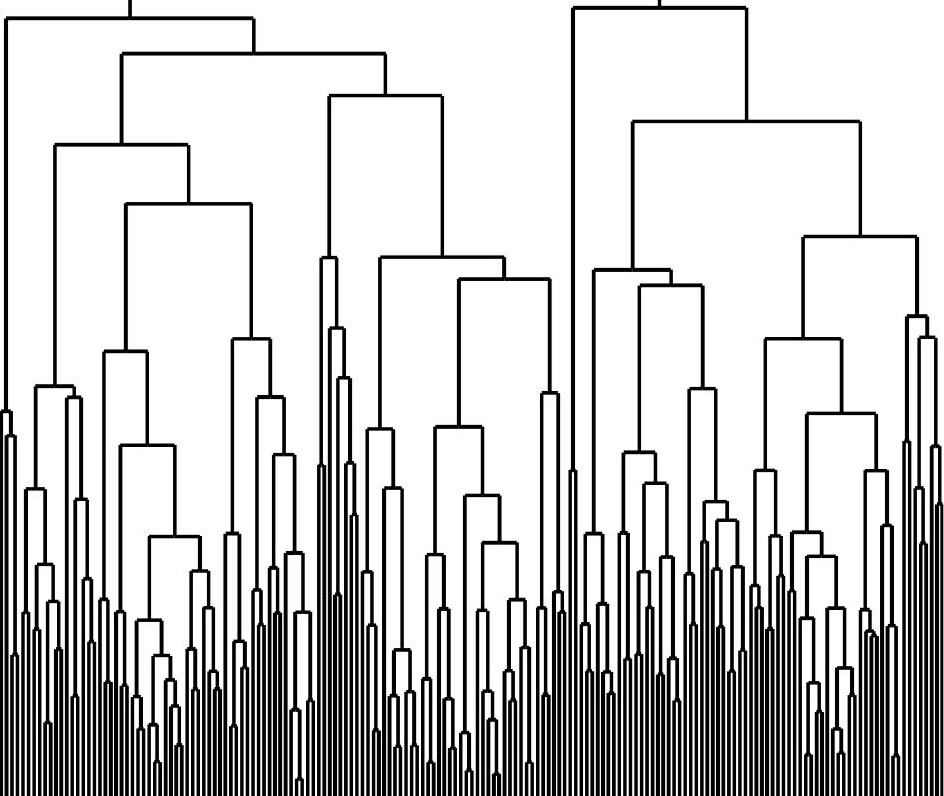

Human Donor

Double Hu-Mice

Antibiotic Treated Hu-Mice

Pre-treatment or Control Hu-Mice 
a

All Samples

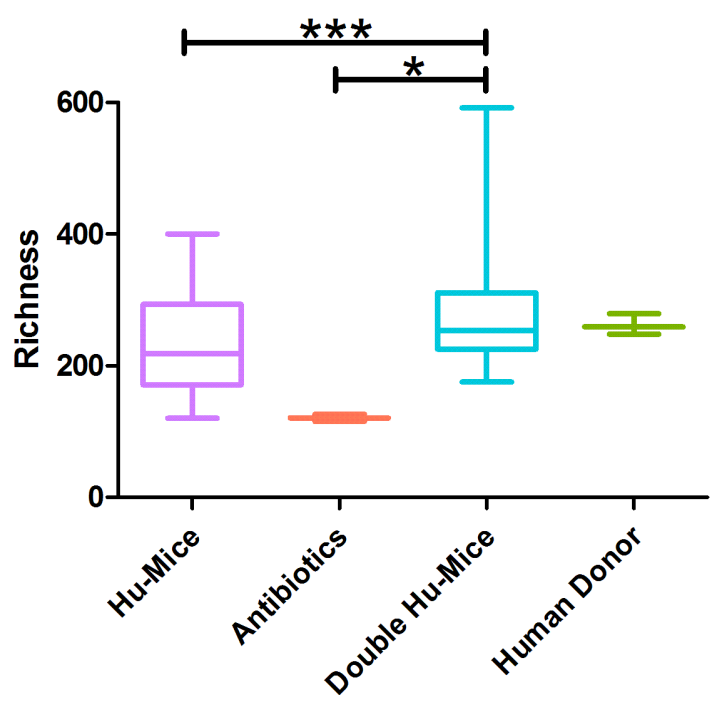

C

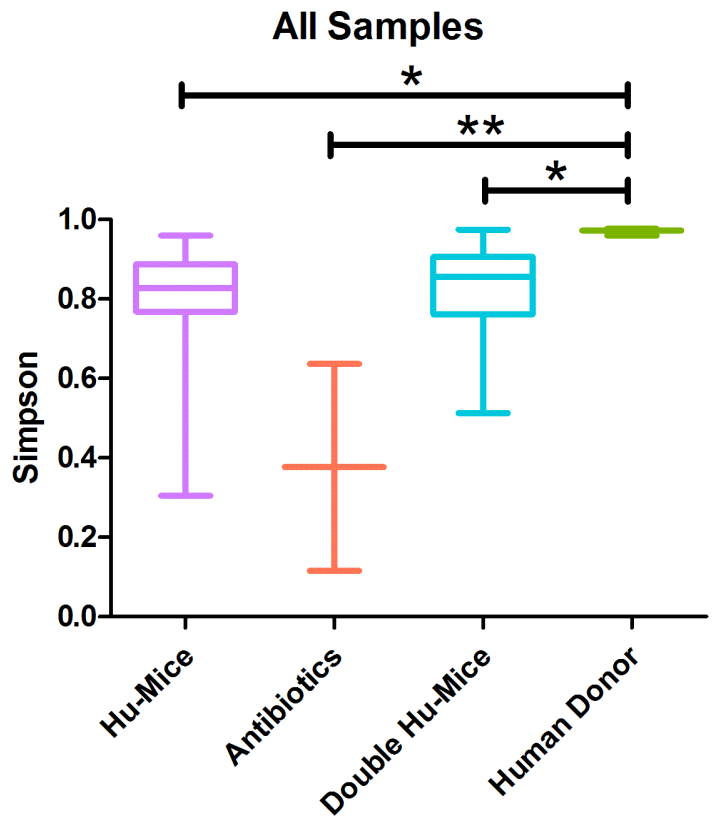

b

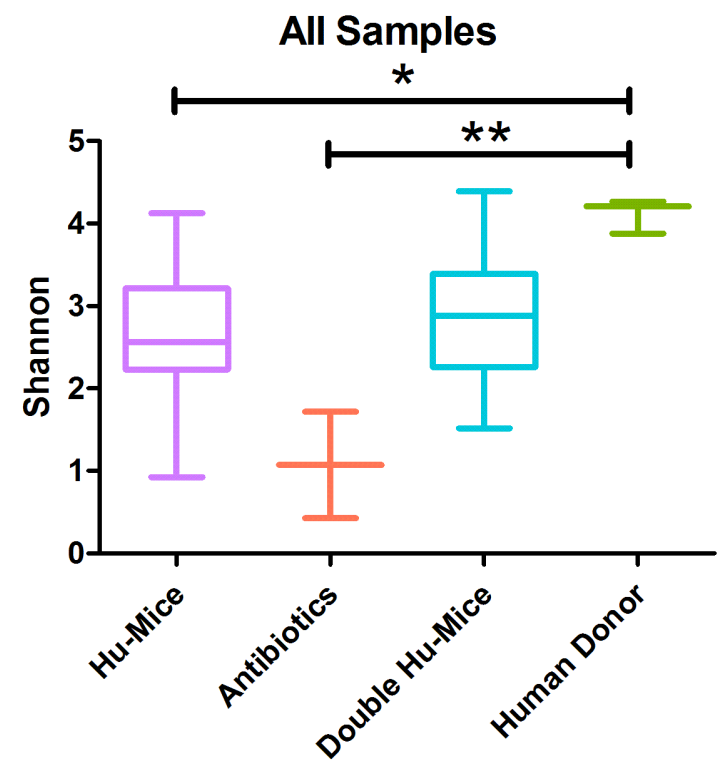




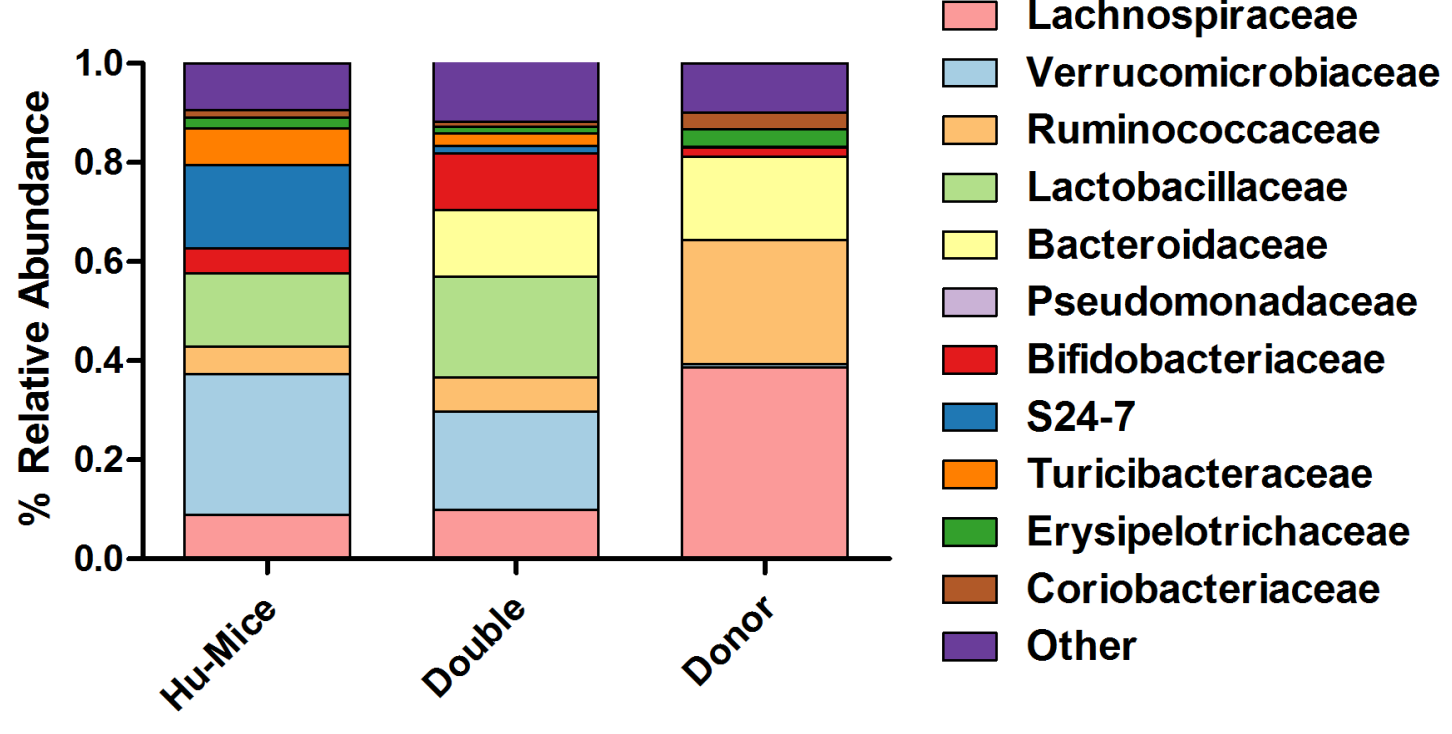


k_Bacteria.p_Firmicutes.c_Bacill

k_Bacteria.p_Firmicutes.c_Bacilli.o_Lactobacillales.f_Lactobacillaceae

k_Bacteria.p_Firmicutes.c_Bacilli.o_Lactobacillales.f_Lactobacillaceae.g_Lactobacillus k_Bacteria.p_Firmicutes.c_Bacilli.o_Lactobacillales.f_Lactobacillaceae.g_Lactobacillus.s_

k_Bacteria.p_Firmicutes.c_Bacilli.o_Lactobacillales

k_Bacteria.p_Verrucomicrobia.c_Verrucomicrobiae.o_Verrucomicrobiales.f_Verrucomicrobiacea

k_Bacteria.p_Verrucomicrobia.c_Verrucomicrobiae.o_Verrucomicrobiales.f_Verrucomicrobiaceae.g_Akkermansia

$k$ Bacteria.p Verrucomicrobia

k_Bacteria.p_Verrucomicrobia.c_Verrucomicrobiae

k_Bacteria.p_Verrucomicrobia.c_Verrucomicrobiae.o_Verrucomicrobiales k_Bacteria.p_Verrucomicrobia.c_Verrucomicrobiae.o_Verrucomicrobiales.f_Verrucomicrobiaceae.g_Akkermansia.s_muciniphila
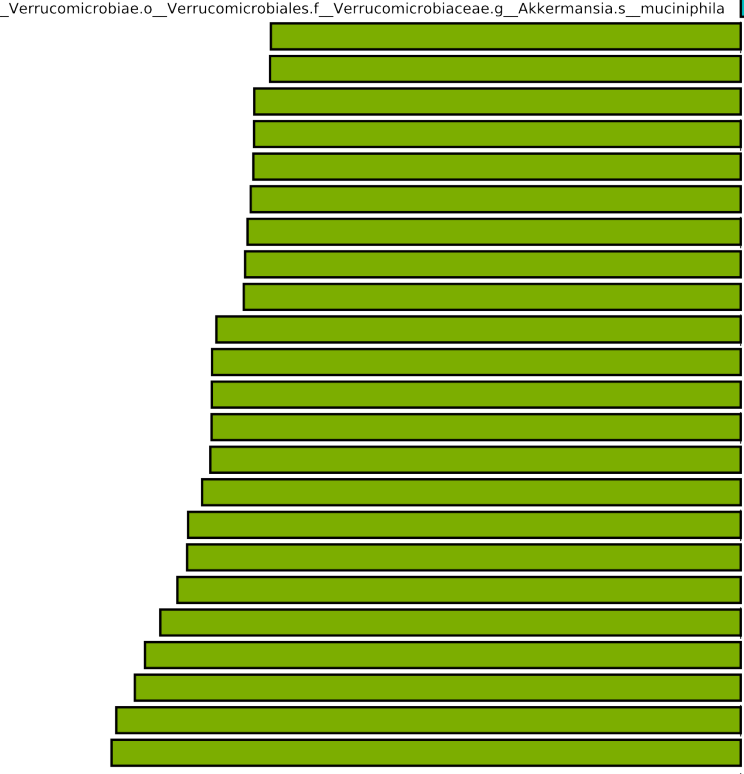

k_Bacteria.p_Firmicutes.c_Clostridia.o_Clostridiales.f_Lachnospiraceae.g__Ruminococcus

k_Bacteria.p_Firmicutes.c_Clostridia.o_Clostridiales.f_Lachnospiraceae.g_Coprococcus.s

k_Bacteria.p_Firmicutes.c_Clostridia.o_Clostridiales.f_Lachnospiraceae.g_Roseburia.s_

k_Bacteria.p_Firmicutes.c_Erysipelotrichi.o_Erysipelotrichales.f_Erysipelotrichaceae.g_Eubacterium_s_biforme

k_Bacteria.p_Actinobacteria.c_Coriobacteriia.o_Coriobacteriales.f_Coriobacteriaceae.g_Collinsella

k_Bacteria.p_Actinobacteria.c_Coriobacteriia.o_Coriobacteriales.f_Coriobacteriaceae.g_Collinsella.s_aerofaciens

k_Bacteria.p_Firmicutes.c_Clostridia.o_Clostridiales.f_Lachnospiraceae.g_

k_Bacteria.p_Firmicutes.c_Clostridia.o_Clostridiales.f_Lachnospiraceae.g_Coprococcus

k_Bacteria.p_Firmicutes.C_Clostridia.o_Clostridiales.f_Lachnospiraceae.g_.s_

k_Bacteria.p_Firmicutes.c_Clostridia.o_Clostridiales.f_Lachnospiraceae.g_Blautia.s_

k_Bacteria.p_Firmicutes.c_Clostridia.o_Clostridiales.f_Ruminococcaceae.

k_Bacteria.p_Firmicutes.c_Clostridia.o_Clostridiales.f_Lachnospiraceae.g_Blautia

k_Bacteria.p_Firmicutes.c_Clostridia.o_Clostridiales.f_Ruminococcaceae.

k_Bacteria.p_Bacteroidetes.c_Bacteroidia.o_Bacteroidales.f_Bacteroidaceae.g_Bacteroides.5_

k_Bacteria.p_Firmicutes.C_Clostridia.o_Clostridiales.f_Lachnospiraceae.g_Roseburia.

k_Bacteria.p_Firmicutes.c_Clostridia.o_Clostridiales.f_Ruminococcaceae.g_Faecalibacterium

k_Bacteria.p_Firmicutes.c_Clostridia.o_Clostridiales.f_Ruminococcaceae.g_Faecalibacterium.s_prausnitzii

k_Bacteria.p_Firmicutes.c_Clostridia.o_Clostridiales.f_Lachnospiraceae.g_Roseburia

k Bacteria.p Firmicutes.c Clostridia.o Clostridiales.f Ruminococcaceae

k_Bacteria.p_Firmicutes

k_Bacteria.p_Firmicutes.C_Clostridia.o_Clostridiales.f_Lachnospiraceae

k_Bacteria.p_Firmicutes.c_Clostridia.o_Clostridiales

k_Bacteria.p_Firmicutes.c_Clostridia

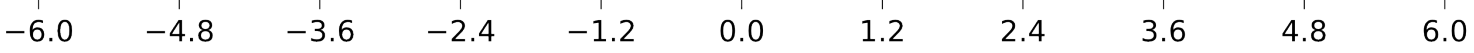

LDA SCORE ( $\log 10)$

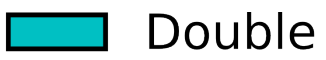

$\square$ Hu-Mice

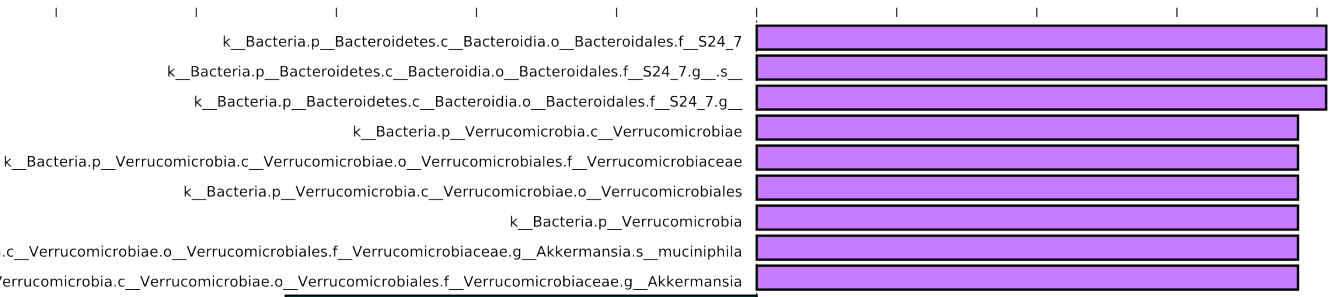

k_Bacteria.p_Bacteroidetes.c_Bacteroidia.o_Bacteroidales.f_Porphyromonadaceae.g_Parabacteroides

k_Bacteria.p_Bacteroidetes.c_Bacteroidia.o_Bacteroidales.f_Porphyromonadaceae

k_Bacteria.p_Bacteroidetes.c_Bacteroidia.o_Bacteroidales.f_Bacteroidaceae.g_Bacteroides.

k_Bacteria.p_Bacteroidetes.c_Bacteroidia.o_Bacteroidales.f_Bacteroidaceae.g_Bacteroides.s_uniformis

k_Bacteria.p_Bacteroidetes.c_Bacteroidia.o_Bacteroidales.f_Bacteroidaceae.g_Bacteroides.s_

k_Bacteria.p_Firmicutes.c_Bacilli.o_Lactobacillales

k_Bacteria.p_Bacteroidetes.c_Bacteroidia.o_Bacteroidales.f_Bacteroidaceae

k_Bacteria.p_Bacteroidetes.c_Bacteroidia.o_Bacteroidales.f_Bacteroidaceae.g_Bacteroides

$\begin{array}{llllll}1 & 1 & & & 1 & 1 \\ -6.0 & -4.8 & -3.6 & -2.4 & -1.2 & 0.0\end{array}$

$1.2 \quad 2.4$

3.6

4.8

6.0 
$a$

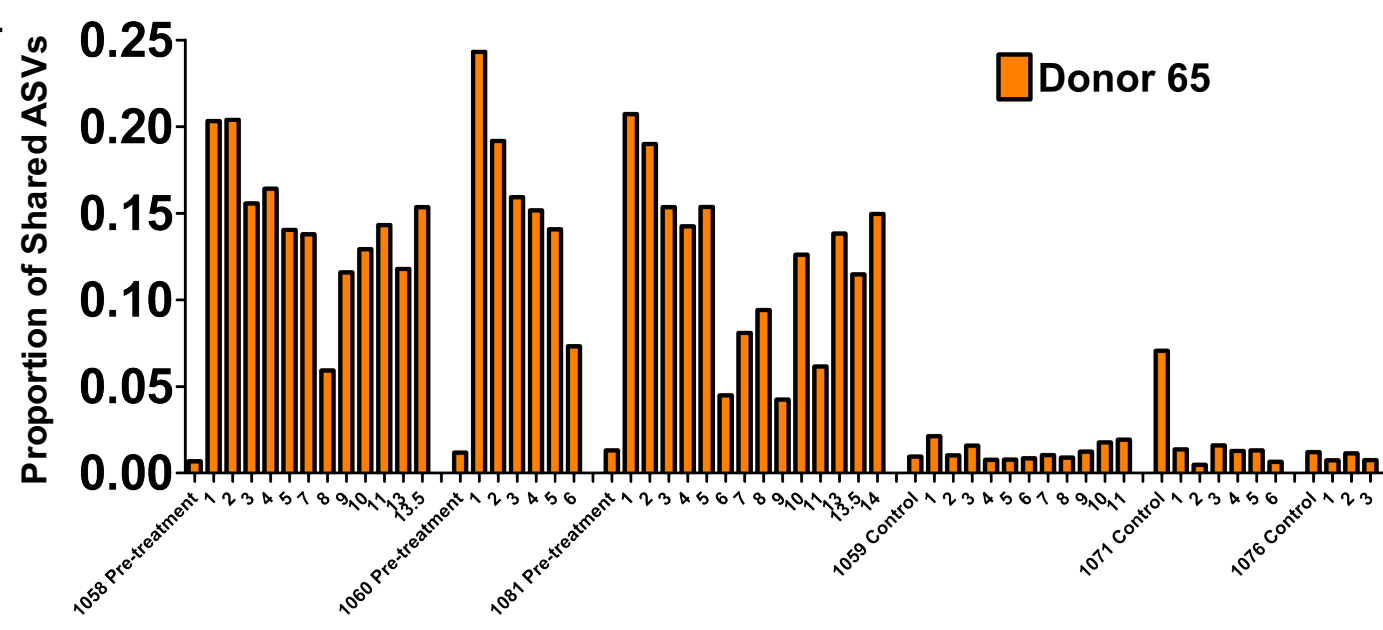

b

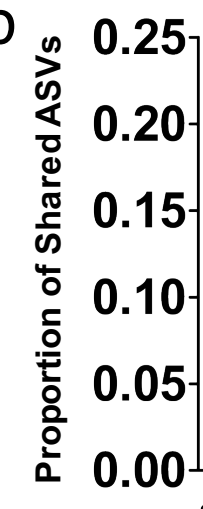

C

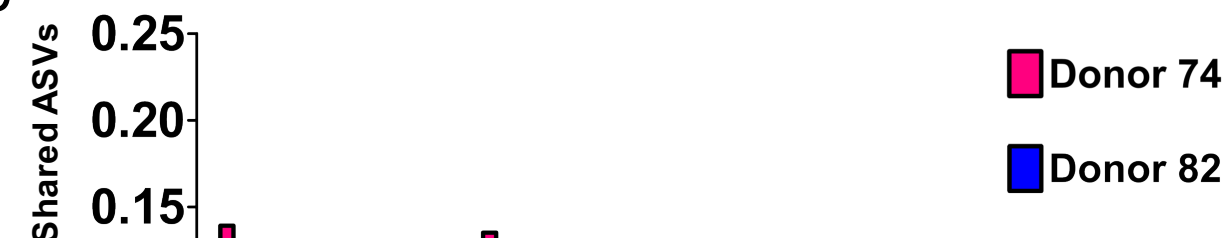

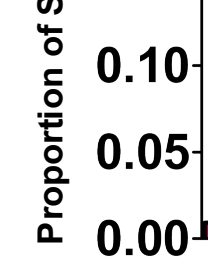

0

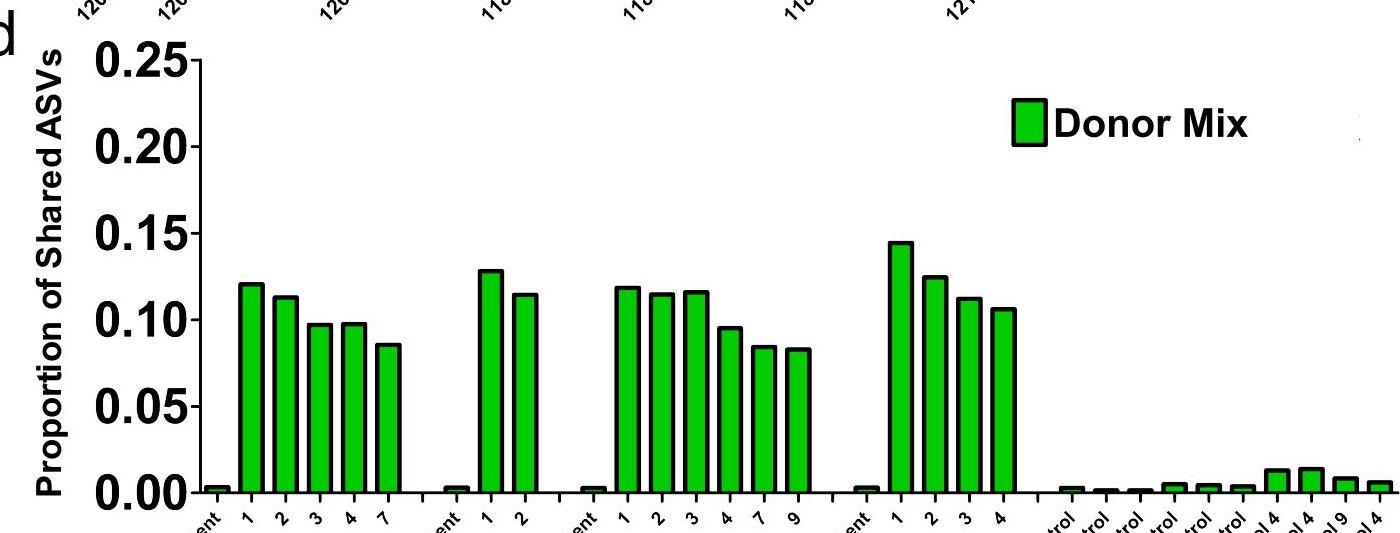


$a$

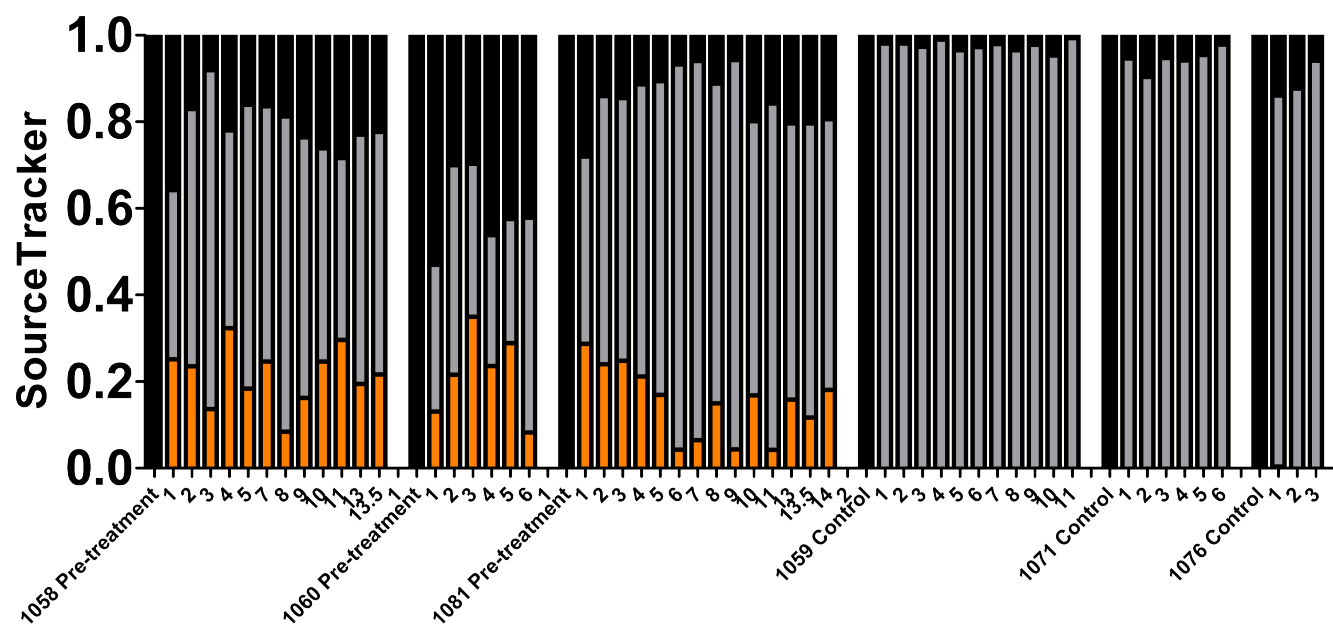

Donor 65

Donor 65

$\square$ Donor 74

Donor 82

Donor Mix

$\square$ Pre-treatment Unknown

b

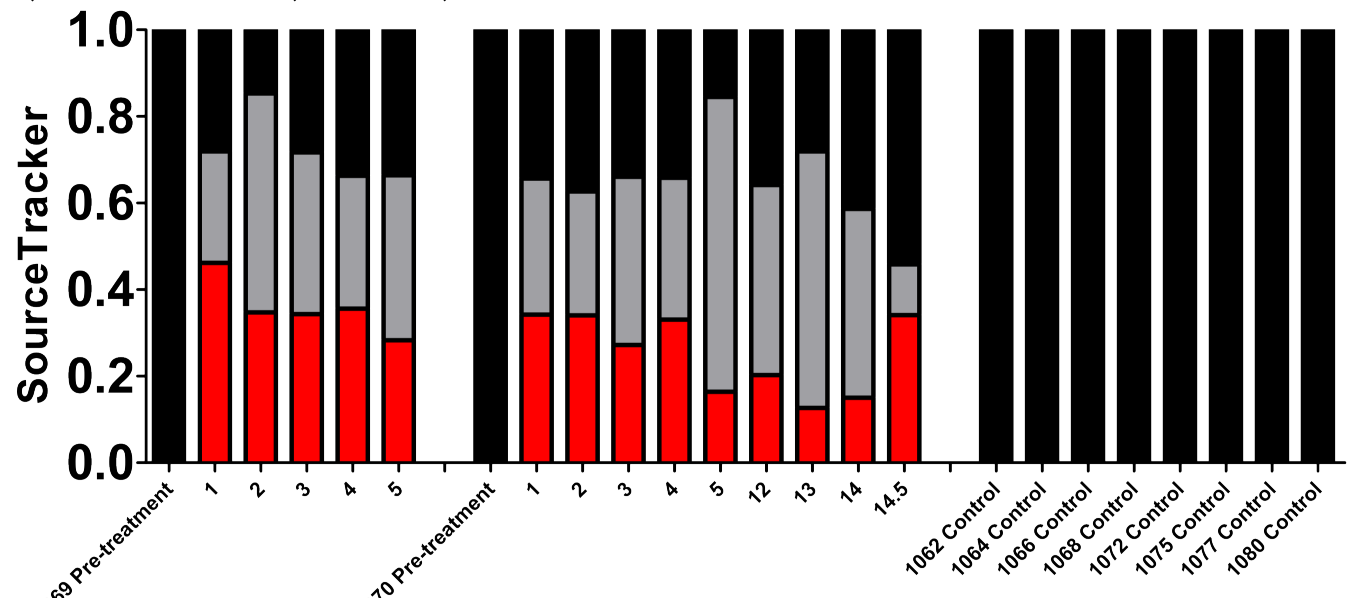

c
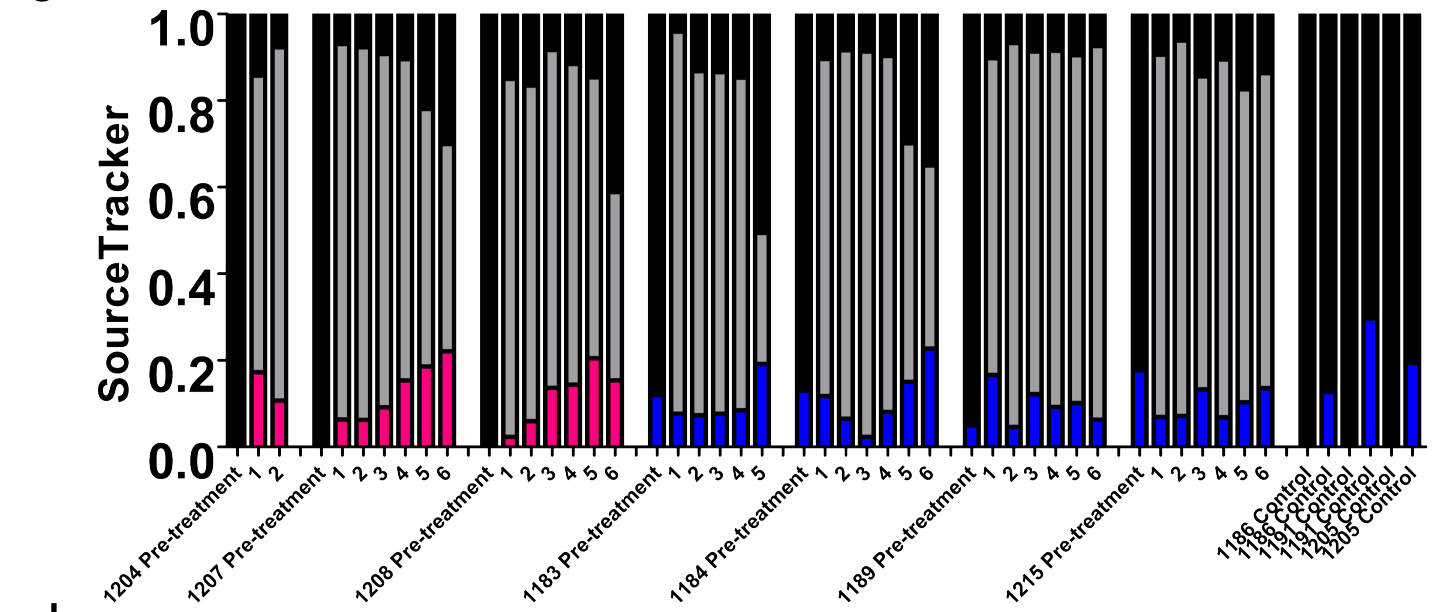

d

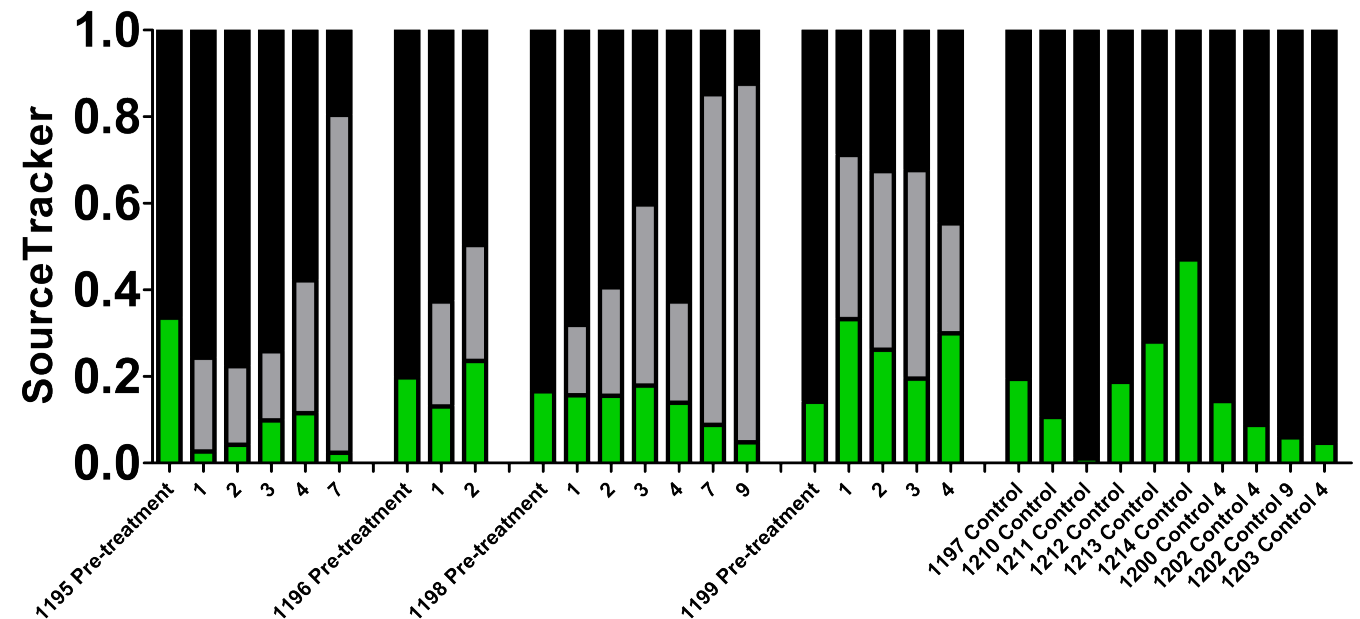


a
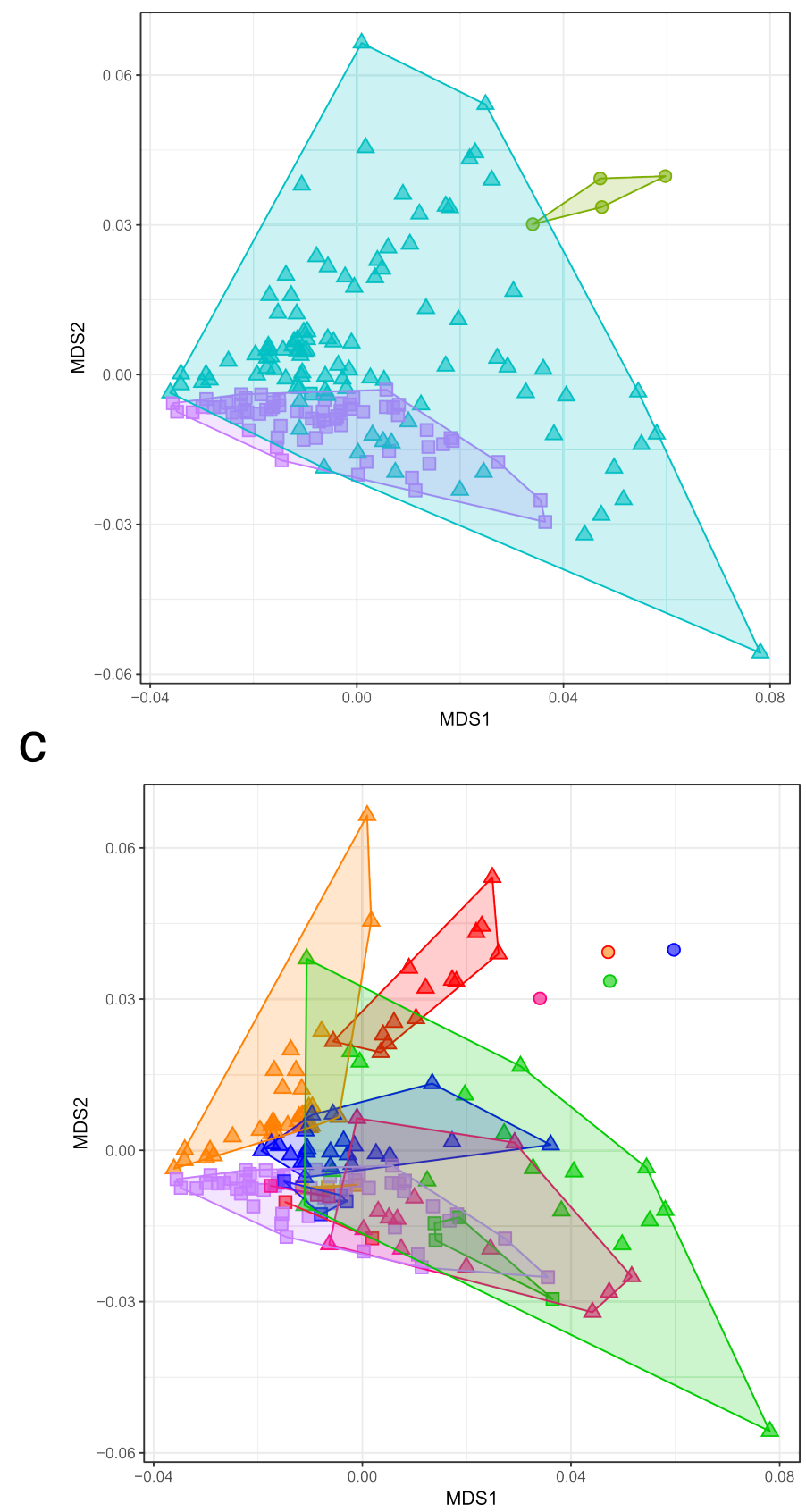

b
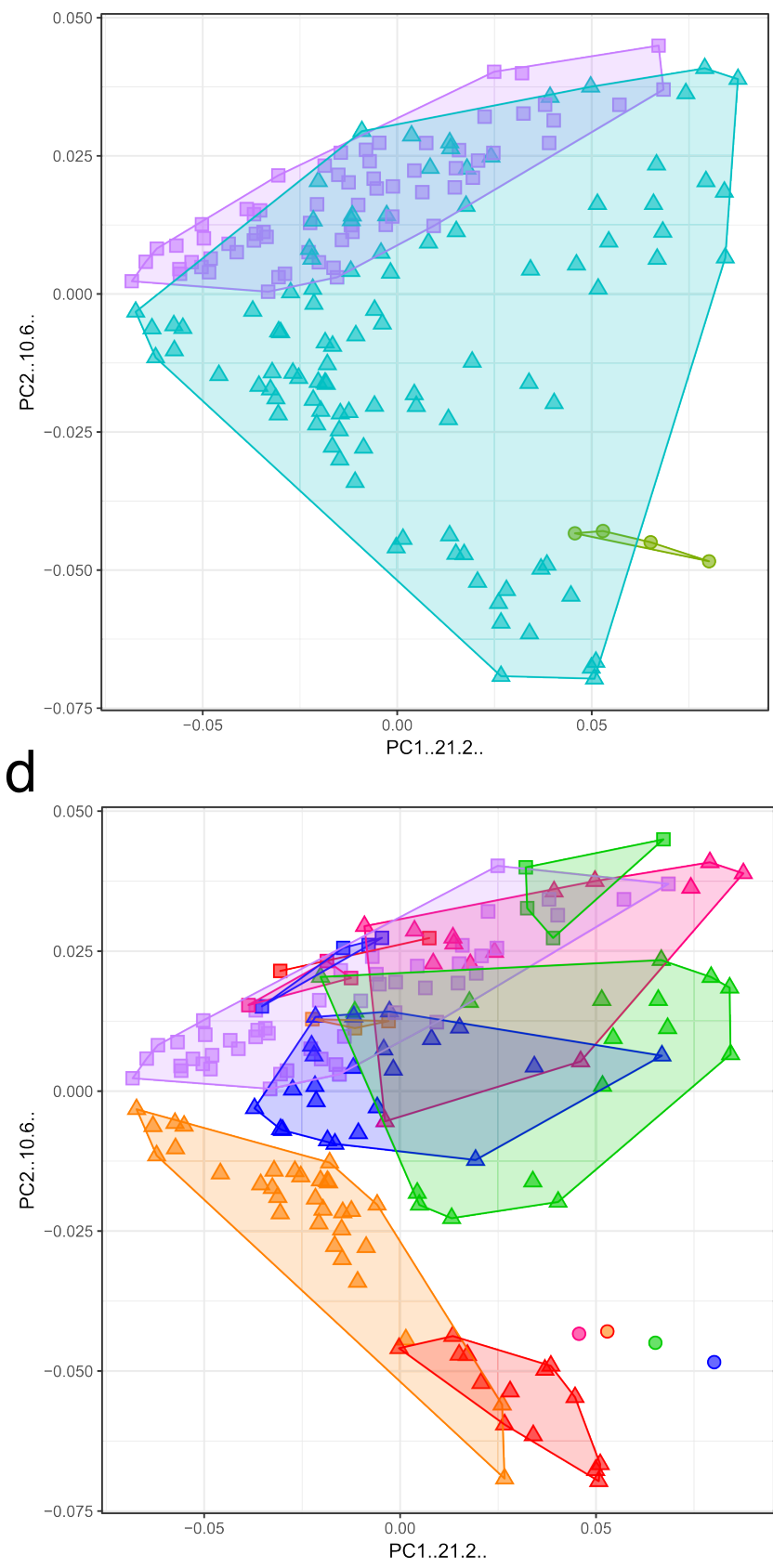

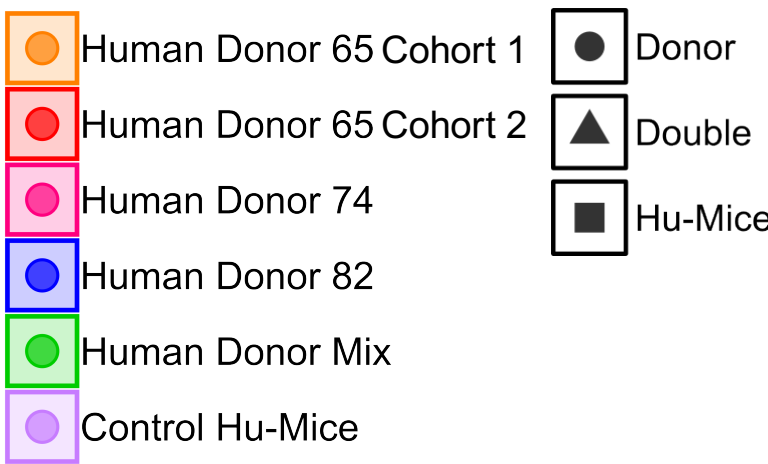

